\title{
Theoretical understanding of unsteady flow separation for shear flow past three square cylinders in vee shape using structural bifurcation analysis
}

\author{
Atendra Kumar ${ }^{1,2} \cdot$ Rajendra K. Ray ${ }^{2}$ (D) \\ Received: 1 July 2019 / Revised: 15 April 2020 / Accepted: 18 May 2020 / Published online: 9 June 2020 \\ (c) SBMAC - Sociedade Brasileira de Matemática Aplicada e Computacional 2020
}

\begin{abstract}
The unsteady flow separation of two-dimensional (2-D) incompressible shear flow past three identical square cylinders arranged in vee shape is studied in this paper, using theoretical structural bifurcation analysis based on topological equivalence. Through this analysis, the exact location and time of occurrence of bifurcation points (flow separation points) associated with secondary and tertiary vortices on all cylinders are studied. The existence of saddle points is also studied during primary flow separation. Different gap ratios between the downstream cylinders, $s / d=0.6-3.0$ (where $s$ is the gap between cylinders, $d$ is the length of cylinder side) with fixed gap $2 d$ between upstream and downstream cylinders for different shear parameter $(K)$ values ranging from $K=0.0$ to 0.4 are considered at Reynolds number (Re) 100. In this process, the instantaneous vorticity contours and streakline patterns, centerline velocity fluctuation, phase diagram, lift and drag coefficients are studied to confirm the theoretical results. Computations are carried out by using higher order compact finite difference scheme. Present study mainly investigates the effect of $K$ and gap ratio on unsteady flow separation and vortex-shedding phenomenon. All the computed results very efficiently and very accurately reproduce the complex flow phenomenon. Through this study, many noticeable and interesting results are reported for the first time for this problem.
\end{abstract}

Keywords Unsteady shear flow · Square cylinders in vee shape · Vortex shedding · Flow separation $\cdot$ HOC scheme $\cdot$ Structural bifurcation

Mathematics Subject Classification 65N06 · 65Z05 · 65Y99

Communicated by Corina Giurgea.

$凶$ Rajendra K. Ray

rajendra@iitmandi.ac.in

Atendra Kumar

atendra.iitd@gmail.com

1 Department of Computational and Data Science, Indian Institute of Science Bangalore, Bangalore, India

2 School of Basic Sciences, Indian Institute of Technology Mandi, Mandi, India 


\section{List of symbols}

$d$

$s$

$\mathrm{Xu}$

$\mathrm{Xd}$

$\mathrm{Yh}$

$U_{\mathrm{c}}$

$\tilde{u}, u$

$\tilde{v}, v$

$\tau$

$t$

$\operatorname{Re}$

$v$

K

G

$x, y$

$\tilde{\psi}, \psi$

$\tilde{\omega}, \omega$

$p$

$C_{\mathrm{L}}$

$C_{\mathrm{D}}$

$P^{*}, T^{*}$

$\emptyset \circ$
Side length of square cylinder

Vertical distance between downstream cylinders

Upstream distance

Downstream distance

Distance to upper and lower boundary from cylinder surface

Centerline velocity

Dimensional and dimensionless horizontal velocities

Dimensional and dimensionless vertical velocities

Dimensional time

Dimensionless time $\left(=\tau U_{\mathrm{c}} / d\right)$

Reynolds number

Kinematic viscosity of the fluid

Non-dimensional shear rate $\left(=G d / U_{\mathrm{c}}\right)$

Dimensional transverse velocity gradient

Cartesian coordinates

Dimensional and dimensionless stream functions

Dimensional and dimensionless vorticities

Dimensionless pressure

Lift coefficient

Drag coefficient

Structural bifurcation point and time

Phase difference between lift coefficients

\section{Introduction}

In many cases of science and engineering practice, the groups of bodies often appear, e.g., groups of buildings, chimneys, stacks, chemical reaction-towers, offshore platforms, etc. Due to the mutual interference, the aerodynamic characteristics, such as pressure distributions and vortex-shedding patterns on each member of the group are completely different from those of an isolated one. Offset-strip and louvered fin exchangers can be approximated as flow around periodic array of individual fin elements. The flow-induced vibration in this type arrangement may not only reduce the life of an equipment, but even lead to serious accidents as well. In the past three decades, most of the research focused on uniform flow around single or multiple square/circular cylinders in various arrangements, but rarely on shear flow around multiple square cylinders. In this paper, a vee-shape arrangement of three square cylinders of the same size in shear flow is investigated as it is one of the most common elements in engineering application. It is well known that flow separation occurs at very early stages of flow past square cylinders due to the sharp corner effect. Therefore, it is very important to understand the mechanism of vortex shedding caused by unsteady flow separation and flowinduced-vibration of each cylinder in the vee-shape arrangement of three square cylinders and flow characteristics around them.

In order to enhance the understanding of the flow phenomenon around three cylinders, the flow phenomenon of flow past two cylinders should also be considered. The experimental study of Zdravkovich (1977, 1987) and Igarashi (1981) on the flow pattern around two cylinders in tandem with different spacing ratios show that the flow characteristics of the cylinders significantly affected by spacing ratios. Mittal et al. (1997) studied the incompressible flow 
past a pair of circular cylinders in tandem and staggered arrangements at Reynolds number 100,1000. The computations are carried out by using a finite element formulation for three different arrangements of cylinders. The results show that flow phenomenon and forces strongly depend on arrangements of cylinders and Reynolds number. An experimental examination of vortex shedding generated by two cylinders arranged in a staggered configuration has been done by Li and Sumner (2009). From the study, the measured Strouhal number is found to be same for closely spaced cylinders, which is the indication of single cylinder behavior. The sound generated by two tandem square cylinders placed in a uniform flow is studied by Inoue et al. (2006). The results show the effect of $s / d$ ratios on the mechanism of the sound generation. The studies discussed till now mainly focused on uniform flow around cylinders. Lankadasu and Vengadesan (2007) studied the interference effect of two square cylinders in tandem in planar shear flow for $R e=100$. The effect of $s / d$ ratio and shear parameter $(K)$ on vortex-shedding phenomenon is discussed in their study. The wake of the upstream cylinder significantly affects the vortex shedding behind the downstream cylinder. These effects vary as a function of $K$ and $s / d$ ratios. Further, several studies on shear flow around single square/circular cylinder is available in the literature (Kiya et al. 1980; Hawang and Sue 1997; Kang 2006; Cheng et al. 2007; Luo et al. 2003; Saha et al. 1999, 2000, 2001; Lankadasu and Vengadesan 2008, 2010; Kumar and Ray 2017). Saha et al. (1999, 2000, 2001) have been given the complete description of effect of inlet shear on the vortex dynamics in cylinder wake. No such study based on shear flow past three square cylinders in the vee-shape arrangement is available in the literature.

Till date, some numerical and experimental investigations of uniform flow around three cylinders have been carried out by a group of researchers. Gu and Sun (2001) study the effect of spacing ratio on flow properties of three cylinders arranged in an equilateral-triangular fashion at $\operatorname{Re}=1.4 \times 10^{4}$ by wind tunnel tests. A numerical simulation of flow past three circular cylinders in a triangular arrangement at low Reynolds number is done by Bao et al. (2010). Computations are done for six gap spacings(s) from 0.5 to 4.0 and for three incidence angles $\alpha=0^{\circ}, 30^{\circ}$ and $60^{\circ}$. The computed results show that the interference effect plays an important role in the variation of the forces and Strouhal number. Moussaoui et al. (2011) study the flow phenomena and heat transfer around three heated square cylinders in vee shape using MRT-D2Q9 model and the MRT-D2Q5 model, respectively. Their investigation considered the fluid as air $(\mathrm{Pr}=0.71)$ and gap-to-diameter ratio from 1 to 2 for Re between 10 and 100. Following this, an experimental informative survey using particle image velocimetry (PIV) technique and dye visualization in an open water channel is carried out for the flow around an equilateral-triangular arrangement of the three spheres investigated by Ozgoren (2013). The experiments show the effect due to the interference of the wake flow, results in more complex flow patterns behind both downstream spheres. The dynamic behavior and convective transport of flow around rows of square cylinders in the staggered arrangement are studied by Chatterjee and Biswas (2015), Chatterjee and Gupta (2015), respectively. Recently, a numerical study of flow around an equilateral triangle of three circular cylinders of equal diameter is done by Zheng et al. (2016). In their study, special attention is paid to the effect of spacing ratios ranging from 1.5 to 7.0 on the variation of flow characteristics among the cylinders at $\operatorname{Re}=100,200$. Three different types of flow patterns are revealed based on the range of spacing ratios.

The primary aim of this paper is to investigate, theoretically as well as numerically, the topological aspects of unsteady flow separation from cylinders surfaces in shear flow. It is well known that topological analysis of flow phenomenon provides guidance for recognition of its structural changes (Kalita and Sen 2013; Ray and Kumar 2017). A thorough analysis of unsteady flow separation for shear flow past single square cylinder using structural bifurcation 
concept can be found in author's recent work (Ray and Kumar 2017). The bifurcations based on topological aspects near a critical point on a stationary plane are studied by Bakker (1989). A survey on new dynamical systems theory related to the topology of 2-D incompressible viscous flows and its application to geophysical fluid dynamics is done by Ma and Wang (2002). Ghil et al. $(2004,2005)$ theoretically studied the topology of the two-dimensional viscous flows near to the solid wall. They use structural bifurcation based boundary layer separation theory and show that a structural bifurcation occurs at a point on the solid boundary whenever a degenerate singularity for vorticity appears on the boundary. The investigations from the topological point of view by considering Taylor's expansion of the velocity field were also carried out for incompressible fluid near a non-simple degenerate critical point close to a stationary wall (Gurcan et al. 2005). Kalita and Sen (2013) study the secondary and tertiary vortex dynamics: sub- $\alpha$ and sub- $\beta$ phenomenon for flow past an impulsively started circular cylinder. Their study describes the interplay among primary, secondary and tertiary vortices for $\operatorname{Re}=5000$, and boundary layer separation corresponding to the secondary and tertiary phenomenon by using topological aspects theoretically.

No such study based on structural bifurcation analysis has been used to analyze unsteady flow separation from cylinders surface for shear flow past three square cylinders in the vee-shape arrangement. In this paper, the effect of the shear parameter on unsteady flow separation leading to vortex formation from the surface of three square cylinders in the veeshape arrangement placed in shear flow is analyzed. The structural bifurcation analysis is used to locate the position and timing of bifurcation points. The stream function-vorticity $(\psi-\omega)$ form of 2-D Navier-Stokes (N-S) equations in Cartesian coordinates is used to compute the flow. The computations have been performed by using higher order compact (HOC) finite difference scheme on uniform Cartesian grids (Kalita et al. 2002). The important points of the present study are to explain the effect of $K$ on unsteady flow separation for different $s / d$ ratios at $\operatorname{Re}=100$.

The paper is organized in the following way. In Sect. 2, we describe the mathematical equations and their discretization procedure. Section 3 deals with results produced and discussion. Finally, In Sect. 4, we summarize our observations in the conclusion part followed by references.

\section{The mathematical equations and its discretization}

The two-dimensional unsteady, incompressible flow of Newtonian fluid past three square cylinders in vee shape is considered here. A schematic diagram of the physical model is shown in Fig. 1. Here, $C_{1}, C_{2}, C_{3}$ are the three square cylinders of same size with $d$ as side length are placed in vee fashion inside a rectangular domain with fixed gap $2 d$ between upstream and downstream cylinders and $s$ is the vertical gap between downstream cylinders. The fluid has non-uniform stream with $U_{\mathrm{c}}$ as centerline velocity. The flow is governed by the incompressible $\mathrm{N}-\mathrm{S}$ equations in stream function-vorticity $(\psi-\omega)$ formulation in Cartesian coordinates $(\tilde{x}, \tilde{y})$; given as

$$
\begin{aligned}
v\left(\frac{\partial^{2} \tilde{\omega}}{\partial \tilde{x}^{2}}+\frac{\partial^{2} \tilde{\omega}}{\partial \tilde{y}^{2}}\right) & =\left(\tilde{u} \frac{\partial \tilde{\omega}}{\partial \tilde{x}}+\tilde{v} \frac{\partial \tilde{\omega}}{\partial \tilde{y}}+\frac{\partial \tilde{\omega}}{\partial \tau}\right), \\
\frac{\partial^{2} \tilde{\psi}}{\partial \tilde{x}^{2}}+\frac{\partial^{2} \tilde{\psi}}{\partial \tilde{y}^{2}} & =-\tilde{\omega} .
\end{aligned}
$$


Here, $\tilde{\psi}$ denotes the stream function, $\tilde{\omega}$ the vorticity, $\tilde{u}, \tilde{v}$ are the horizontal and vertical velocity components, respectively, $\tau$ is the time and $v$ the kinematic viscosity. The velocity components $\tilde{u}$ and $\tilde{v}$ are related to stream function $\tilde{\psi}$ as

$$
(\tilde{u}, \tilde{v})=\left(\frac{\partial \tilde{\psi}}{\partial \tilde{y}}, \frac{\partial \tilde{\psi}}{\partial \tilde{x}}\right)
$$

and the vorticity as

$$
\tilde{\omega}=\frac{\partial \tilde{v}}{\partial \tilde{x}}-\frac{\partial \tilde{u}}{\partial \tilde{y}} .
$$

All quantities in the above equations and relations are dimensional, their non-dimensional forms are given by

$$
x=\frac{\tilde{x}}{d}, y=\frac{\tilde{y}}{d}, t=\frac{\tau U_{\mathrm{c}}}{d}, \psi=\frac{\tilde{\psi}}{U_{\mathrm{c}} d}, \omega=\frac{\tilde{\omega} d}{U_{\mathrm{c}}}, u=\frac{\tilde{u}}{U_{\mathrm{c}}}, v=\frac{\tilde{v}}{U_{\mathrm{c}}} .
$$

So, the non-dimensional form of above Eqs. (1) and (2) becomes

$$
\begin{aligned}
& \frac{\partial^{2} \omega}{\partial x^{2}}+\frac{\partial^{2} \omega}{\partial y^{2}}=\operatorname{Re}\left(u \frac{\partial \omega}{\partial x}+v \frac{\partial \omega}{\partial y}+\frac{\partial \omega}{\partial t}\right), \\
& \frac{\partial^{2} \psi}{\partial x^{2}}+\frac{\partial^{2} \psi}{\partial y^{2}}=-\omega .
\end{aligned}
$$

Now, the dimensionless velocities $u, v$ in terms of dimensionless stream function are written as

$$
(u, v)=\left(\frac{\partial \psi}{\partial y}, \frac{\partial \psi}{\partial x}\right),
$$

whereas the dimensionless vorticity $\omega$ is given by

$$
\omega=\frac{\partial v}{\partial x}-\frac{\partial u}{\partial y} .
$$

To compute the pressure $(p)$, we have used the dimensionless pressure Poisson equation given below

$$
\nabla^{2} p=2\left(\frac{\partial u}{\partial y} \frac{\partial v}{\partial x}-\frac{\partial u}{\partial x} \frac{\partial v}{\partial y}\right)
$$

Now, we will describe the boundary conditions associated with Eqs. (3), (4) and (5). At inlet, we consider a linear shear velocity profile, i.e.:

$$
u=U_{\mathrm{c}}+K y, v=0,
$$

where $K$ denotes the shear rate and is defined as $K=G d / U_{\mathrm{c}}$, where $G$ is the dimensional velocity gradient in vertical direction. At the outlet boundary, a convective boundary condition, i.e.:

$$
\frac{\partial \phi}{\partial t}+U_{\mathrm{c}} \frac{\partial \phi}{\partial x}=0
$$

is used (Lankadasu and Vengadesan 2007). No-slip conditions are considered on the surfaces of all the cylinders; whereas slip boundary condition is used on the top and bottom boundaries 


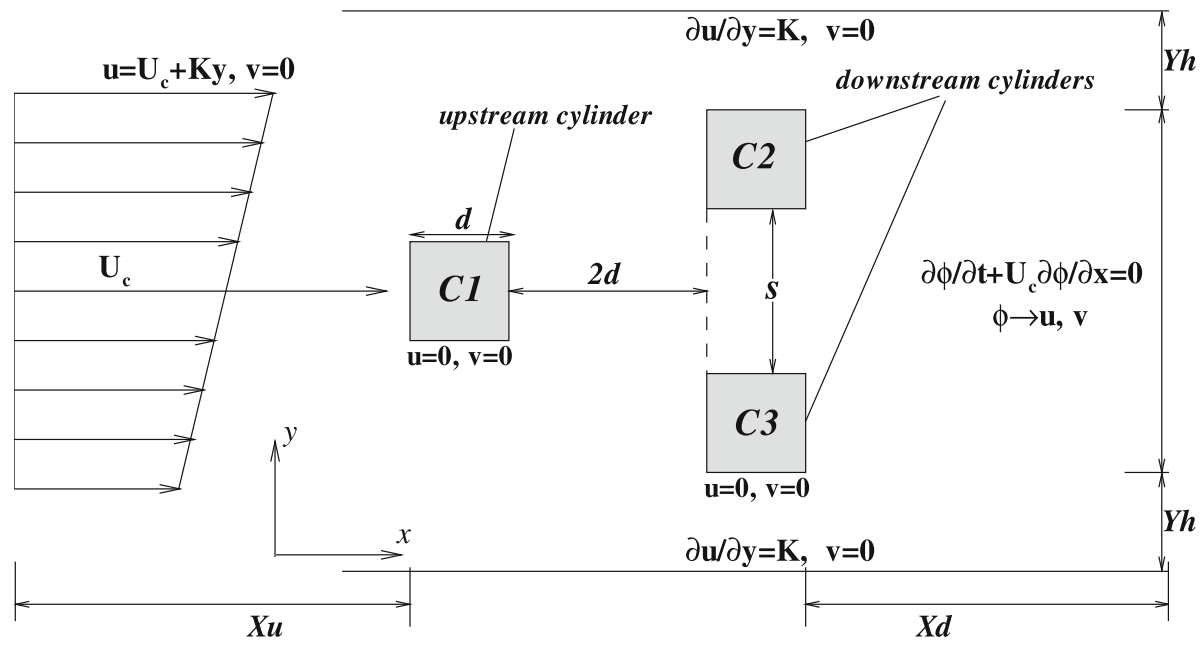

Fig. 1 Schematic diagram of the flow past three square cylinders in vee-shape arrangement

of the computational domain (Ray and Kumar 2017) given as

$$
\frac{\partial u}{\partial y}=K, v=0 .
$$

The boundary conditions for pressure are considered as the zero-gradient condition on the above boundaries. In the $\psi-\omega$ formulation used here, the stream function values on the surface of the cylinders are zero $(\psi=0)$.

Additionally, we may use Eq. (4) and above conditions to obtain the following vorticity condition on the surfaces of each cylinder:

$$
\begin{aligned}
& \omega=-\frac{\partial^{2} \psi}{\partial x^{2}} \text { On the left and right boundaries of the cylinder } \\
& \omega=-\frac{\partial^{2} \psi}{\partial y^{2}} \quad \text { On the upper and lower boundaries of the cylinder }
\end{aligned}
$$

\subsection{Numerical discretization}

To discretize the governing Eqs. (3) and (4), we use the transformation-free HOC finite difference scheme Kalita et al. (2002) on uniform Cartesian grids. The scheme is 2nd order accurate in time and 4th order accurate in space. The formulation is successfully used in our previous works to simulate shear flow past single square cylinder (Kumar and Ray 2015, 2016; Ray and Kumar 2017; Kumar and Ray 2018) and two cylinders in parallel arrangement (Kumar and Ray 2017), for circular cylinder (Ray and Kalita 2010). For the discretization of equations, we construct a uniform Cartesian mesh in the rectangular region $\left[x_{1}, x_{2}\right] \times\left[y_{1}, y_{2}\right]$ by the grid points $\left(x_{i}, y_{j}\right)$. The HOC discretization of Eqs. (3) and (4) at the $(i, j)$ th grid point of the computational domain is given as

$$
\begin{aligned}
& {\left[\operatorname{Re}+A 11_{i j} \delta_{x}{ }^{2}+A 12_{i j} \delta_{y}{ }^{2}+A 13_{i j} \delta_{x}+A 14_{i j} \delta_{y}+A 15_{i j} \delta_{x} \delta_{y}\right.} \\
& \left.+A 16_{i j} \delta_{x} \delta_{y}{ }^{2}+A 17_{i j} \delta_{x}{ }^{2} \delta_{y}+A 18_{i j} \delta_{x}{ }^{2} \delta_{y}{ }^{2}\right] \omega_{i j}^{n+1}
\end{aligned}
$$




$$
\begin{aligned}
= & {\left[\operatorname{Re}+A 21_{i j} \delta_{x}{ }^{2}+A 22_{i j} \delta_{y}{ }^{2}+A 23_{i j} \delta_{x}+A 24_{i j} \delta_{y}+A 25_{i j} \delta_{x} \delta_{y}\right.} \\
& \left.+A 26_{i j} \delta_{x} \delta_{y}{ }^{2}+A 27_{i j} \delta_{x}{ }^{2} \delta_{y}+A 28_{i j} \delta_{x}{ }^{2} \delta_{y}{ }^{2}\right] \omega_{i j}^{n},
\end{aligned}
$$

and

$$
\left[\delta_{x}^{2}+\delta_{y}^{2}-(H 2+K 2) \delta_{x}^{2} \delta_{y}^{2}\right] \psi_{i j}=\left[-1+H 2 \delta_{x}^{2}+K 2 \delta_{y}{ }^{2}\right], \omega_{i j},
$$

respectively, where the coefficients are defined as

$$
\begin{aligned}
& A 11_{i j}=-H 12 \operatorname{Re}-0.5 \Delta t A 1_{i j}, A 21_{i j}=-H 12 \operatorname{Re}+0.5 \Delta t A 1_{i j}, \\
& A 12_{i j}=-K 12 \operatorname{Re}-0.5 \Delta t A 2_{i j}, A 22_{i j}=-K 12 \operatorname{Re}+0.5 \Delta t A 2_{i j}, \\
& A 13_{i j}=-H 11 \operatorname{Re}-H 12 u_{i j} \operatorname{Re}^{2}-0.5 \Delta t A 3_{i j}, \\
& A 23_{i j}=-H 11 \operatorname{Re}-H 12 u_{i j} \operatorname{Re}^{2}+0.5 \Delta t A 3_{i j}, \\
& A 14_{i j}=-K 11 \operatorname{Re}-K 12 v_{i j} \operatorname{Re}^{2}-0.5 \Delta t A 4_{i j}, \\
& A 24_{i j}=-K 11 \operatorname{Re}-K 12 v_{i j} \operatorname{Re}^{2}+0.5 \Delta t A 4_{i j}, \\
& A 15_{i j}=-0.5 \Delta t A 5_{i j}, A 25_{i j}=0.5 \Delta t A 5_{i j}, \\
& A 16_{i j}=-0.5 \Delta t A 6_{i j}, A 26_{i j}=0.5 \Delta t A 6_{i j}, \\
& A 17_{i j}=-0.5 \Delta t A 7_{i j}, A 27_{i j}=0.5 \Delta t A 7_{i j}, \\
& A 18_{i j}=-0.5 \Delta t A 8_{i j}, A 28_{i j}=0.5 \Delta t A 8_{i j},
\end{aligned}
$$

where

$$
\begin{aligned}
A 1_{i j}= & 1+H 11 \operatorname{Re} u_{i j}+H 12 \operatorname{Re}^{2} u_{i j}^{2}+2 H 12 \operatorname{Re}\left(u_{x}\right)_{i j}, \\
A 2_{i j}= & +K 11 \operatorname{Re} v_{i j}+K 12 \operatorname{Re}^{2} v_{i j}^{2}+2 K 12 \operatorname{Re}\left(v_{y}\right)_{i j} \\
A 3_{i j}= & -\operatorname{Re} u_{i j}+H 11 \operatorname{Re}\left(u_{x}\right)_{i j}+K 11 \operatorname{Re}\left(u_{y}\right)_{i j}+H 12 \operatorname{Re}^{2} u_{i j}\left(u_{x}\right)_{i j} \\
& +H 12 \operatorname{Re}\left(u_{x x}\right)_{i j}+K 12 \operatorname{Re}\left(u_{y y}\right)_{i j}+K 12 \operatorname{Re}^{2} v_{i j}\left(u_{y}\right)_{i j}, \\
A 4_{i j}= & -\operatorname{Re} v_{i j}+H 11 \operatorname{Re}\left(v_{x}\right)_{i j}+K 11 \operatorname{Re}\left(v_{y}\right)_{i j}+H 12 \operatorname{Re}^{2} u_{i j}\left(v_{x}\right)_{i j} \\
& +H 12 \operatorname{Re}\left(v_{x x}\right)_{i j}+K 12 \operatorname{Re}\left(v_{y y}\right)_{i j}+K 12 \operatorname{Re}^{2} v\left(v_{y}\right)_{i j}, \\
A 5_{i j}= & H 11 \operatorname{Re} v_{i j}+K 11 \operatorname{Re} u_{i j}+H 12 \operatorname{Re}^{2} u_{i j} v_{i j}+2 H 12 \operatorname{Re}\left(v_{x}\right)_{i j} \\
& +2 K 12 \operatorname{Re}\left(u_{y}\right)_{i j}+K 12 \operatorname{Re} u_{i j} v_{i j}, \\
A 6_{i j}= & -H 11-H 12 \operatorname{Re} u_{i j}+K 12 \operatorname{Re} u_{i j} \\
A 7_{i j}= & -K 11+H 12 \operatorname{Re} v_{i j}-K 12 \operatorname{Re} v_{i j}, \\
A 8_{i j}= & -H 12-K 12, \\
H 2= & -\frac{h^{2}}{12}, \quad K 2=-\frac{h^{2}}{12} \\
H 11= & \operatorname{Reu}_{i j} \frac{h^{2}}{6}, \quad K 11=\operatorname{Re} v_{i j} \frac{h^{2}}{6} \\
H 12= & -\frac{h^{2}}{12}, \quad K 12=-\frac{h^{2}}{12} .
\end{aligned}
$$

The details of the finite difference operators $\delta_{x}, \delta_{x}^{2}, \delta_{y}$ and $\delta_{y}^{2}$ can be found in Appendix 1 . In brief, the discretization procedure of the governing differential equations leading to the system of linear algebraic Eqs. (7), (8) can be found in Kalita et al. (2002). 
The numerical approximation of the boundary conditions for stream function, velocities and pressure are straightforward. We need to approximate the vorticity boundary condition on the surface of the square cylinders. The HOC approximation of vorticity conditions on the surface of the cylinder given in Eq. (6) are as follows:

At the left wall of the upstream square cylinder, consider the index for $x$ is $L$, we have

$$
\left.\omega\right|_{L, j}=-\left.\frac{\partial^{2} \psi}{\partial x^{2}}\right|_{L, j}
$$

Employing a Taylor series expansion, we get

$$
v_{L, j}=-\left.\frac{\partial \psi}{\partial x}\right|_{L, j}=-\delta_{x} \psi_{L, j}+\left.\frac{h}{2} \frac{\partial^{2} \psi}{\partial x^{2}}\right|_{L, j}+\left.\frac{h^{2}}{6} \frac{\partial^{3} \psi}{\partial x^{3}}\right|_{L, j}+\left.\frac{h^{3}}{24} \frac{\partial^{4} \psi}{\partial x^{4}}\right|_{L, j}+O\left(h^{4}\right) .
$$

As $v_{L, j}=0$, using (9) in (10), we have

$$
0=-\delta_{x}^{-} \psi_{L, j}-\frac{h \omega_{L, j}}{2}-\left.\frac{h^{2}}{6} \frac{\partial \omega}{\partial x}\right|_{L, j}-\left.\frac{h^{3}}{24} \frac{\partial^{2} \omega}{\partial x^{2}}\right|_{L, j}+O\left(h^{4}\right) .
$$

Making use of the fact that on the cylinder surface $u=0, v=0$, Eq. (1) gives,

$$
\left.\frac{\partial^{2} \omega}{\partial x^{2}}\right|_{L, j}=\left.\operatorname{Re} \frac{\partial \omega}{\partial t}\right|_{L, j}-\left.\frac{\partial^{2} \omega}{\partial y^{2}}\right|_{L, j} .
$$

By using (12) in (11), we get

$$
0=-\delta_{x}^{-} \psi_{L, j}-\frac{h \omega_{L, j}}{2}+\left.\left(\frac{h^{3}}{24}-\frac{h^{2}}{6}\right) \frac{\partial \omega}{\partial x}\right|_{L, j}-\left.\frac{h^{3} \operatorname{Re}}{24} \frac{\partial \omega}{\partial t}\right|_{L, j}+\left.\frac{h^{3}}{24} \frac{\partial^{2} \omega}{\partial y^{2}}\right|_{L, j} .
$$

Using forward difference for the time derivative and second-order backward difference $\left(\delta^{-}\right)$ for the derivatives along $x$-direction, we finally get

$$
\begin{aligned}
\omega_{L, j}^{n+1}= & \frac{24 \Delta t}{h^{3} \operatorname{Re}}\left[\left\{\frac{h^{3} \operatorname{Re}}{24 \Delta t}-\frac{h^{2}}{16}-\frac{h}{3}\right\} \omega_{L, j}^{n}+\frac{h}{24} \omega_{L, j-1}^{n}+\frac{h}{24} \omega_{L, j+1}^{n}\right. \\
& \left.+\left(\frac{h^{2}}{24}-\frac{h}{6}\right)\left(2 \omega_{L-1, j}^{n}-\frac{1}{2} \omega_{L-2, j}^{n}\right)\right] .
\end{aligned}
$$

\section{Results and discussion}

\subsection{Grid independence}

We first perform the grid independence study for variation of the grid on both space and time increments for $\operatorname{Re}=100, K=0.1$. The values of $u, v, \psi, \omega$ at a monitoring point $(0.74,-0.5)$ behind the upstream cylinder at two time levels, $t=1.0,10.0$, on four different grid sizes $(401 \times 138),(501 \times 172),(801 \times 276)$, and $(1601 \times 551)$ are shown in Table 1 . As one can see from the table that grid size $(801 \times 276)$ is enough to produce reasonable results. It is clear that the variables $u, v, \psi, \omega$ vary approximately by $1.5 \%$ for the grid sizes $(801 \times 276)$, $(1601 \times 551)$. As such, the next study for time increment independence was performed on a spatial grid $(801 \times 276)$ by considering the time increments $\Delta t=0.001,0.005$ and 0.01 . In Table 2, we show the variable values at the same monitoring point $(0.74,-0.5)$ behind the upstream cylinder for three time increments. The variable values for $\Delta t=0.001,0.01$ show a difference of $2.1 \%$ approximately. From above observations, it is clear that the grid 
Table 1 Stream function, vorticity, pressure and velocity values at a monitoring point $(0.74,-0.5)$ behind the upstream cylinder for $s / d=1.0$ for different grid sizes with $\Delta t=0.01$ at $\operatorname{Re}=100, K=0.1$

\begin{tabular}{lllllll}
\hline$t$ & $(I \times J)$ & $u$ & $v$ & $\psi$ & $\omega$ & $p$ \\
\hline 1.0 & $(401 \times 138)$ & 0.5912 & 0.2876 & -0.2874 & -0.0872 & 0.5241 \\
& $(501 \times 172)$ & 0.6454 & 0.3578 & -0.1982 & -0.1254 & 0.6134 \\
& $(801 \times 276)$ & 0.6718 & 0.3822 & -0.1880 & -0.1327 & 0.6802 \\
& $(1601 \times 551)$ & 0.6824 & 0.3902 & -0.1848 & -0.1304 & 0.6924 \\
10.0 & $(401 \times 138)$ & 0.2752 & -0.8214 & 0.0984 & 3.6521 & 0.7452 \\
& $(501 \times 172)$ & 0.3178 & -0.7342 & 0.1346 & 4.1272 & 0.8104 \\
& $(801 \times 276)$ & 0.3387 & -0.7174 & 0.1444 & 4.3546 & 0.8573 \\
& $(1601 \times 551)$ & 0.3445 & -0.7045 & 0.1468 & 4.4421 & 0.8742 \\
\hline
\end{tabular}

Table 2 The effect of time increment on the results at $R e=100, K=0.1, s / d=1.0$

\begin{tabular}{lllllll}
\hline$\Delta t$ & $(I \times J)$ & $u$ & $v$ & $\psi$ & $\omega$ & $p$ \\
\hline 0.001 & $(801 \times 276)$ & 0.3342 & -0.7142 & 0.1432 & 4.3203 & 0.8506 \\
0.005 & $(801 \times 276)$ & 0.3361 & -0.7102 & 0.1439 & 4.3354 & 0.8545 \\
0.01 & $(801 \times 276)$ & 0.3387 & -0.7174 & 0.1444 & 4.3546 & 0.8573
\end{tabular}

The values of $u, v, \psi, \omega, p$ are taken at point $(0.74,-0.5)$ at time $t=10.0$

$(801 \times 276)$ and $\Delta t=0.01$ is sufficient to capture the flow phenomena accurately. So, for further calculations, we consider grid of size $(801 \times 276)$ on domain size $7 d-5 d-29 d$ (where $7 d$ is the distance from inlet boundary to upstream cylinder front surface, $5 d$ is the distance from upstream cylinder top surface to top boundary of domain and $29 d$ is from upstream cylinder rear surface to outflow boundary) with $\Delta t=0.01$.

A detailed description of unsteady flow separation for shear flow past single square cylinder can be found in the author's previous work (Ray and Kumar 2017). The present study is concerned with the unsteady flow separation leading to interesting vortex dynamics from the interaction of all three square cylinders in the vee-shape arrangement and interplay among them. The computations are carried out for different values of $K$ ranging from 0.0 to 0.4 , with different vertical gap distances between the downstream cylinders in the range, $s / d=$ $0.6-3.0$, for $\operatorname{Re}=100$. The study includes influence due to the variation of the shear parameter and gap distance.

As the title of our paper suggests, we are interested in finding exact position and timing of flow separation using structural bifurcation analysis from cylinders surfaces. In this process, first we want to investigate the instantaneous flow phenomenon in terms of vorticity, streakline patterns and lift-drag coefficients for fully developed flow.

\subsection{Instantaneous flow phenomenon for $s / d=0.6$}

The instantaneous vorticity contours for $s / d=0.6$ with different $K$ values are shown in Fig. 2 for fully developed flow. It is observed that the flow behind all square cylinders eventually becomes almost steady state for all $K$ values. For $K=0.0$, the wake is symmetric about centerline $(y=0)$ and a pair of elongated vortices are developed in the downstream as shown in Fig. 2a. The vortices are no longer distinct and some of them are seen to be 


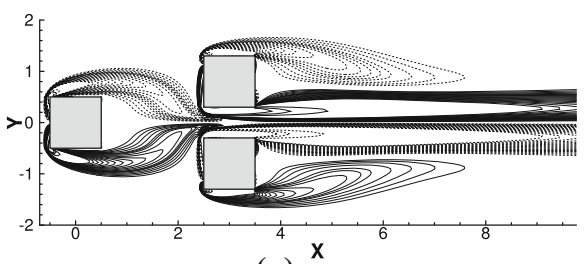

(a)

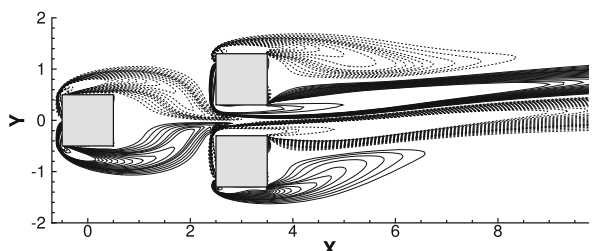

(b)

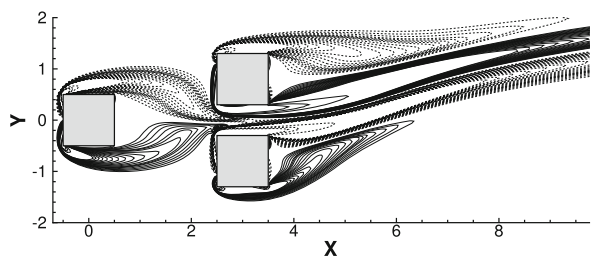

(d)

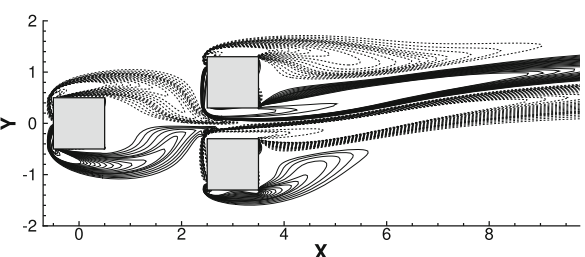

(c)

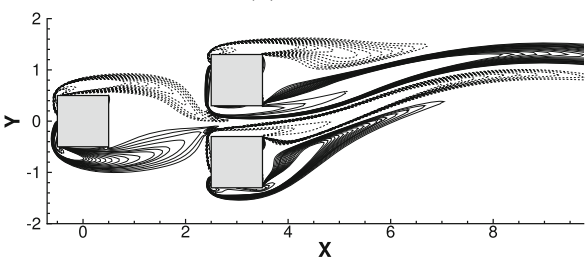

(e)

Fig. 2 Vorticity contours for fully developed flow at $\operatorname{Re}=100, s / d=0.6$, solid line contours represent positive vortex, dotted line contours represent negative vortex a $K=0.0$, b $K=0.1$, c $K=0.2, \mathbf{d} K=0.3$, e $K=0.4$

substantially smaller than the normally shed vortices. This can be attributed to the fact that the flow interference between downstream cylinders is quite strong at this small vertical gap between the cylinders and consequently oscillations occur in the wake. A flip-flopping pattern is seen behind the downstream cylinders. With increasing shear rate, the flow behavior changes significantly as shown in Fig. 2b-e. The flow behind the upstream cylinder develops with the inception of two almost symmetric vortices for all $K$ values, whereas the formation of steady vortices behind the downstream cylinders ' $\mathrm{C} 2$ ' and ' $\mathrm{C} 3$ ' have the tendency to move away from the line of symmetry $(y=0)$ for non-zero $K$. The corresponding pattern of instantaneous streaklines for $s / d=0.6$ are shown in Fig. 3 for all $K$ values.

The most important parameters for flow past bluff bodies to understand the flow properties are the lift and drag coefficients. Figures 4 and 5 plot the temporal variation of lift and drag coefficients for $s / d=0.6$ and $K=0.0-0.4$. These figures clearly show almost steady-state behavior of the flow for all $K$ values.

In Fig. 6, the fluctuation of $u$-velocity along $x=0$ line, $v$-velocity along $y=0$ line for different $K$ values are shown. In the case of $K=0.0$, the flow accelerates equally on both sides of the square cylinder. For $K>0.0$, the acceleration of the flow on the upper part (highvelocity side) of the cylinder and on the lower part (low-velocity side) of the cylinder are not the same. It is also seen that $v$-velocity fluctuates with the larger amplitude of oscillations along $x$-axis in near wake only. This indicates that the effect of cylinders on the flow is not significant in far wake for this Re value. The similar flow behavior is seen for $s / d=1.0$ for all $K$ values. 


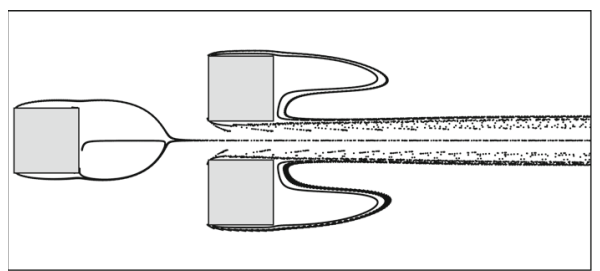

(a)

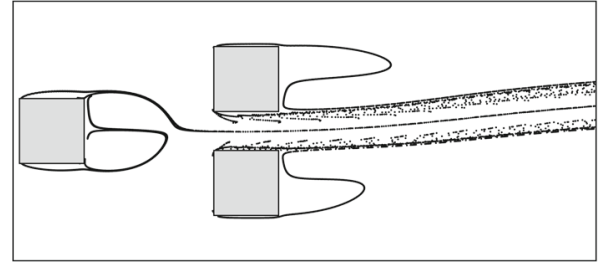

(b)

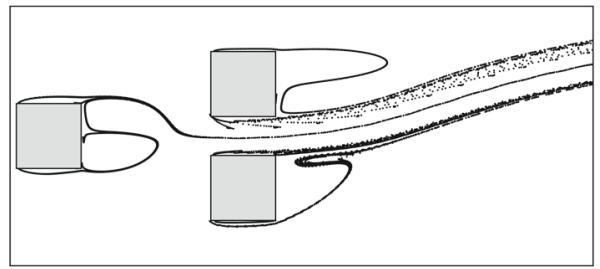

(d)

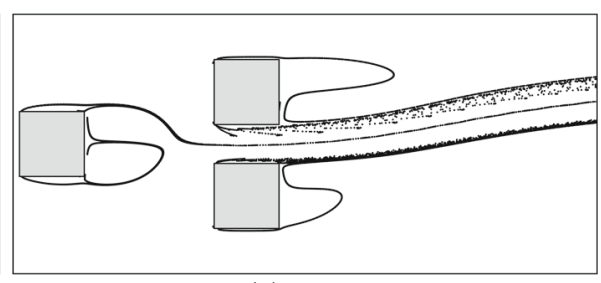

(c)

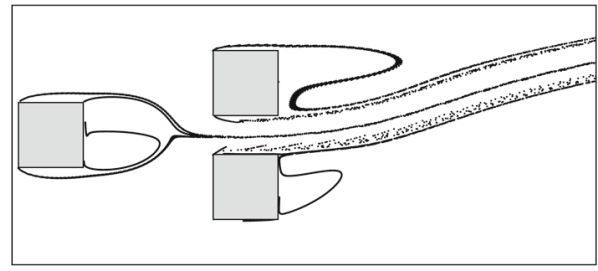

(e)

Fig. 3 Streakline patterns for $\operatorname{Re}=100, s / d=0.6$, a $K=0.0, \mathbf{b} K=0.1$, c $K=0.2$, d $K=0.3$, e $K=0.4$

\subsection{Instantaneous flow phenomenon for $s / d=\mathbf{2 . 0}$}

Further increase in the vertical spacing between downstream square cylinders, $s / d$, to 2.0 the flow in the gap achieves enough strength to influence the vortex-shedding phenomenon (see Fig. 7). The flow behind the cylinders ' $\mathrm{C} 2$ ' and ' $\mathrm{C} 3$ ' become unsteady from initial and the vortices of opposite sign are developed alternately from rear surface of each cylinder for all $K$ values.

The flow through the gap of the down stream cylinders (named as gap flow) disturbs the simple movement of vortices in the wake region of the downstream cylinders and develops a periodic vortex shedding in the downstream. One can also see that for $K=0.0$, there is almost no crossing of fluid flow across the centerline. The wake behind both the downstream cylinders ' $\mathrm{C} 2$ ' and 'C3' is termed as anti-phase synchronized pattern (Chatterjee et al. 2009; Mittal et al. 2017) with phase difference $188^{\circ}$ (see Fig. 7a). For a non-zero value of $K$, the vortices shed from each cylinder crossover centerline and interact with each other. At $K=0.1$, the wake still shows anti-phase synchronized pattern with smaller phase difference $131^{\circ}$ (Fig. 7b) between 'C2' and 'C3' cylinders. As $K$ increases, the vortex formation from the lower side of downstream cylinder ' $\mathrm{C} 3$ ' is fully suppressed (Fig. 7d, e). The frequency of the vortices shed from the cylinder ' $\mathrm{C} 2$ ' is significantly differed by the frequency of vortices shed behind the cylinder ' $\mathrm{C} 3$ ' with increasing $K$ values. The size and strength of positive and negative vortices differ significantly. We observe that in all the cases vortices generated from 'C2' and 'C3' are in anti-phase with the phase difference decreasing with increasing 


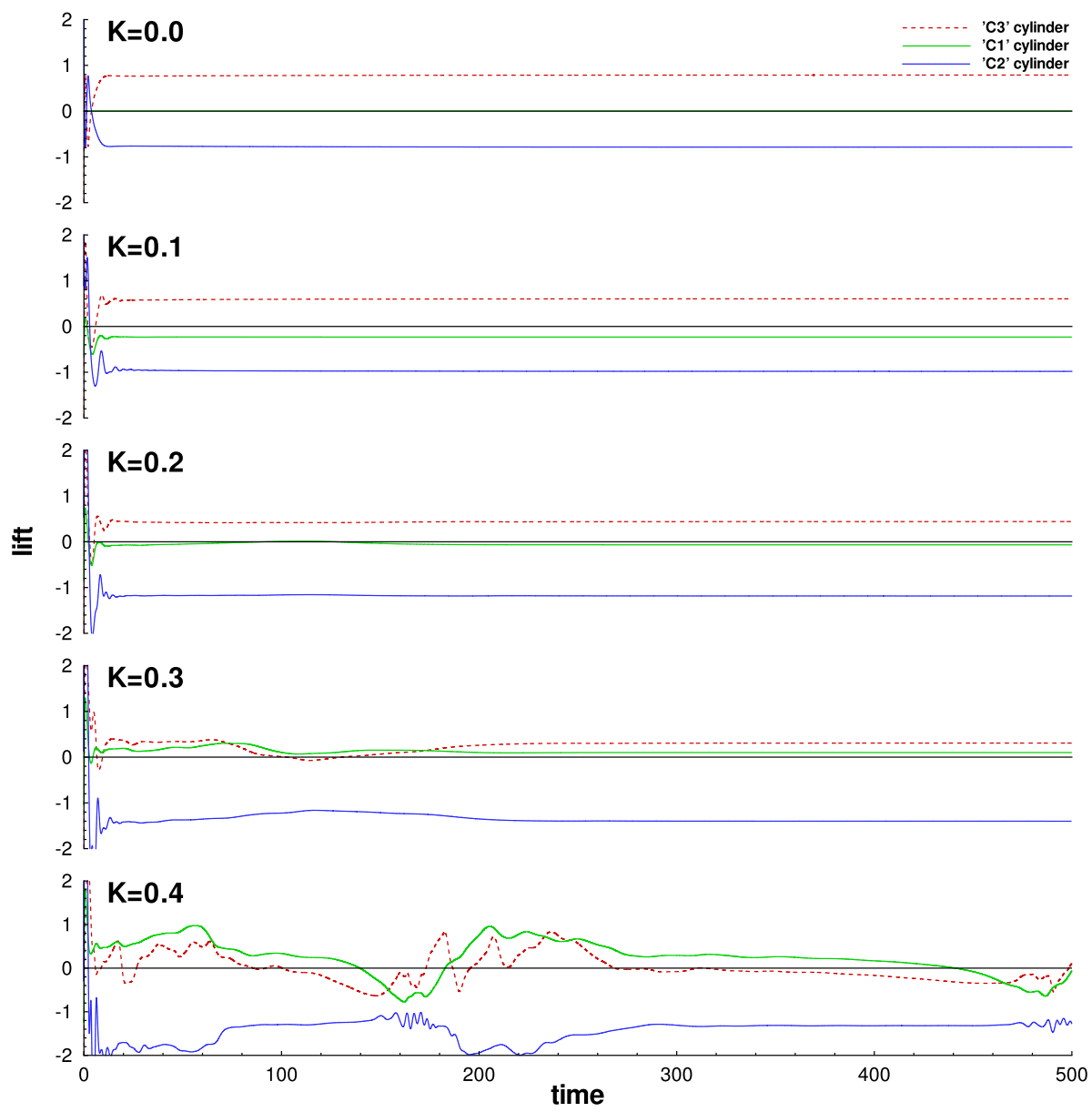

Fig. 4 Lift coefficient fluctuation with time for $s / d=0.6$

$K$ values considered here, as shown in the Table 3. Similar observations are also made from the patterns of streaklines shown in Fig. 8. In Fig. 9, we plot the axial velocity profiles for $u$ vs $y$ at $x=0.0$ and $v$ vs $x$ at $y=0.0$. From the figure, it is clear that, as $K$ increases, $u$ increases in the upper part and decrease in the lower part of the cylinder, whereas the amplitude of fluctuation of $v$ is more in near wake region behind the downstream cylinders. The observations described above from the vortex-shedding phenomenon can be further verified by the plots of lift (Fig. 10) and drag coefficients (Fig. 11). Figures 10 and 11 clearly depict the sinusoidal behavior of lift and drag coefficients for all parameters with the different amplitudes of oscillations. It clearly depicts that there is a definite phase difference between the signals of lift coefficients corresponding to ' $\mathrm{C} 2$ ' and ' $\mathrm{C} 3$ ' cylinders (Fig. 10). One can see that the amplitude of oscillation of both coefficients for cylinder ' $\mathrm{C} 2$ ' gradually increases with increasing $K$ value, which also confirms the vortex shedding from ' $\mathrm{C} 2$ '. The frequency of oscillations decreases with increasing $K$. The phase diagrams by considering values of $u, v$ at a monitoring point $(3.74,-2.0)$ for all values of $K$ are plotted in Fig. 12 for all $K$ values. The figure clearly depicts the periodic nature of the flow. 


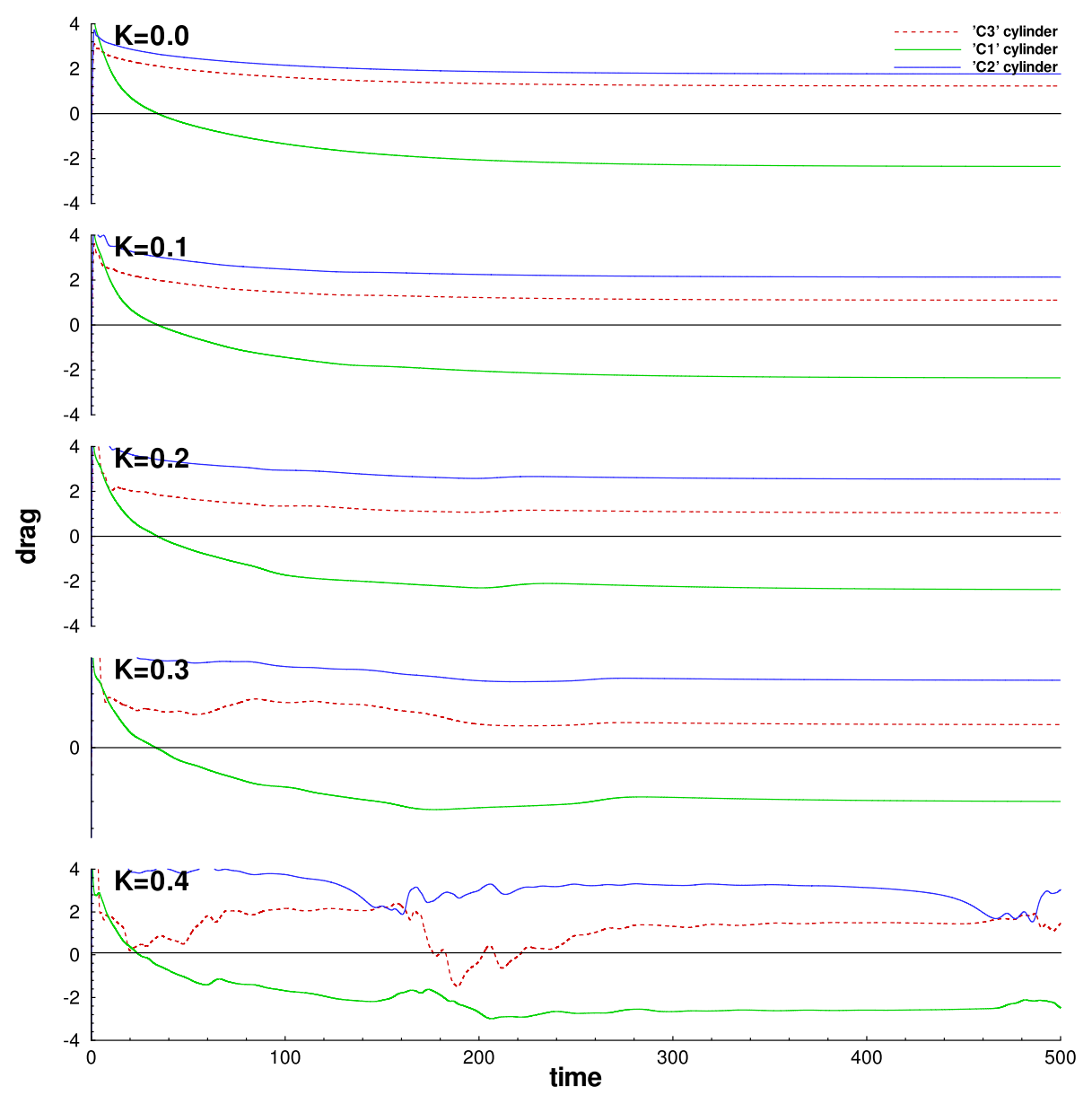

Fig. 5 Drag coefficient fluctuation with time for $s / d=0.6$

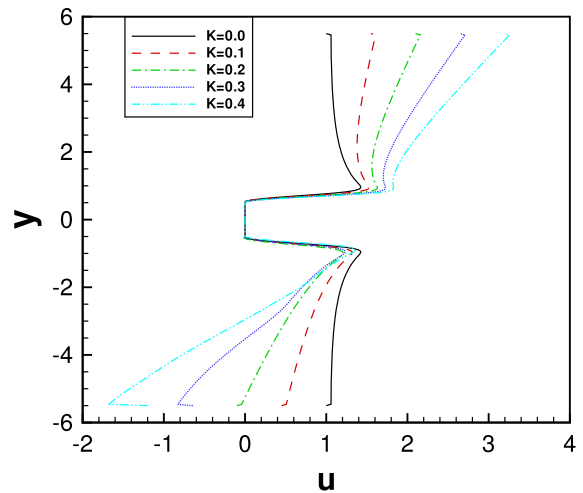

(a)

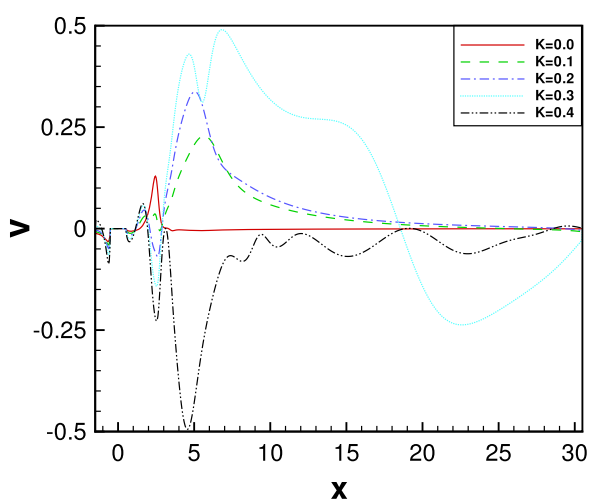

(b)

Fig. 6 Center-line velocity fluctuation for $\operatorname{Re}=100, s / d=0.6$ at different $K$ values a $u$ along $y$-axis $\mathbf{b} v$ along $x$-axis 


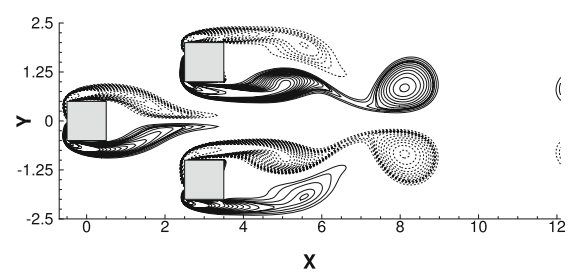

(a)

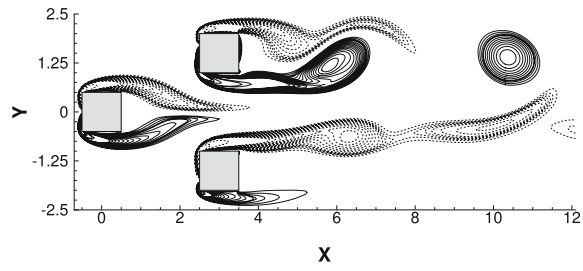

(b)

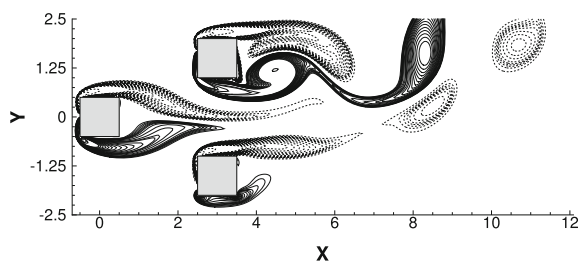

(d)

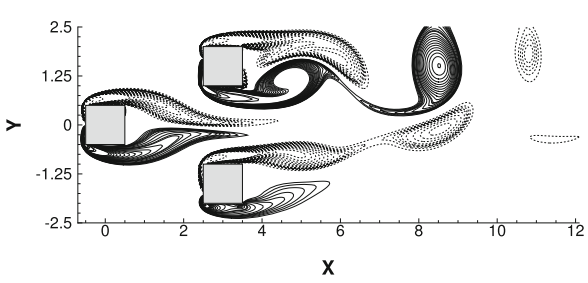

(c)

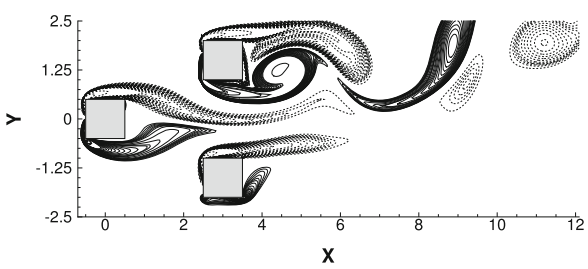

(e)

Fig. 7 Vorticity contours for fully developed flow at $\operatorname{Re}=100, s / d=2.0$, solid line contours represent positive vortex, dotted line contours represent negative vortex a $K=0.0$, b $K=0.1$, c $K=0.2, \mathbf{d} K=0.3$, e $K=0.4$

Table 3 Variation of phase difference between downstream cylinders with $s / d$ and $K$

\begin{tabular}{llllll}
\hline$(s / d) \backslash(K)$ & 0.0 & 0.1 & 0.2 & 0.3 & 0.4 \\
\hline 2.0 & $188^{\circ}$ & $131^{\circ}$ & $99^{\circ}$ & $75^{\circ}$ & $69^{\circ}$ \\
3.0 & $185.5^{\circ}$ & $172^{\circ}$ & $159^{\circ}$ & $147^{\circ}$ & $153^{\circ}$ \\
\hline
\end{tabular}

\subsection{Instantaneous flow phenomenon for $\mathrm{s} / \mathrm{d}=\mathbf{3 . 0}$}

With further increase in the value of $s / d$ to 3.0, the vortices shed behind the cylinders are clearly apparent (Fig. 13); however, there are some evidence of vortex merging for a nonzero value of $K$ in the downstream. The vortices are separately visible throughout the domain for $K=0.0$, this is attributed to the weakly interactive flow pattern for this gap between the cylinders. The vortex shedding from the cylinder ' $\mathrm{C} 2$ ' seems to have a definite phase relationship with the shedding from the cylinder ' $\mathrm{C} 3$ '. As a results, the anti-phase shedding of vortices from ' $\mathrm{C} 2$ ' and ' $\mathrm{C} 3$ ' is predominant for $K=0.0$ with the quantitative phase difference has the value $185.5^{\circ}$ (Fig. 13a). When $K$ increases, the vortices interact with each other by crossing the centerline. A pair of elongated vortices form behind the upstream cylinder (' $\mathrm{C} 1$ ') and spreads sideways while being restricted by the small gap between the cylinders ' $\mathrm{C} 2$ ' and ' $\mathrm{C} 3$ '. As $K$ increases, the interference of vortices behind downstream cylinders is complicated by incident shear velocity profile, results in an asymmetry about 


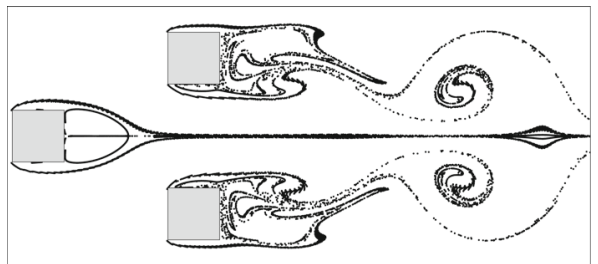

(a)

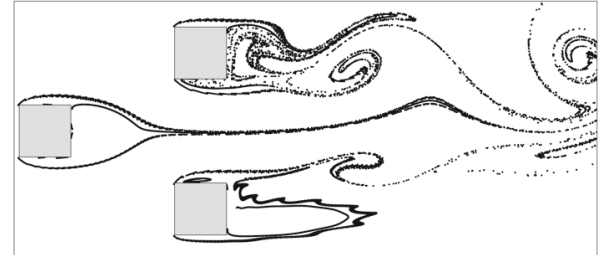

(b)

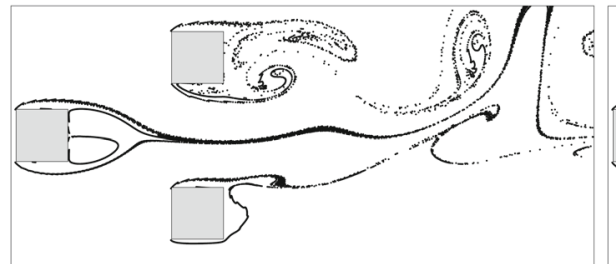

(d)

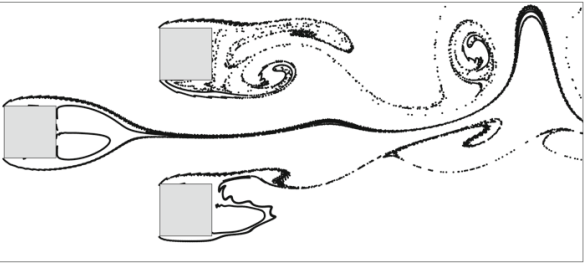

(c)

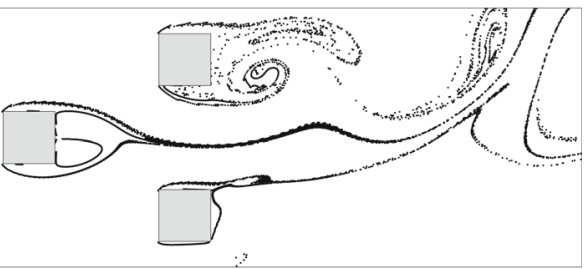

(e)

Fig. 8 Streakline patterns for Re $=100, s / d=2.0$, a $K=0.0$, b $K=0.1$, c $K=0.2$, d $K=0.3$, e $K=0.4$

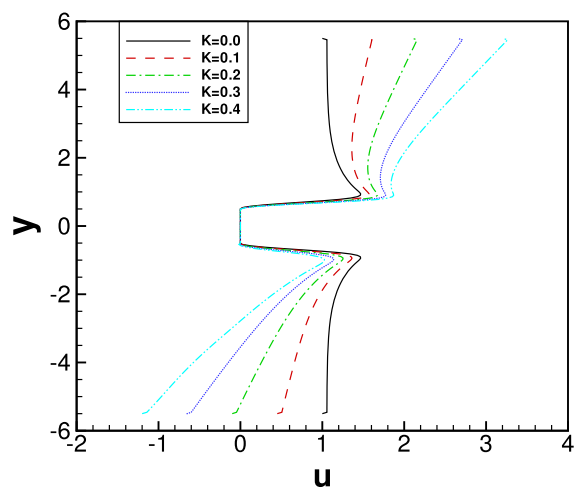

(a)

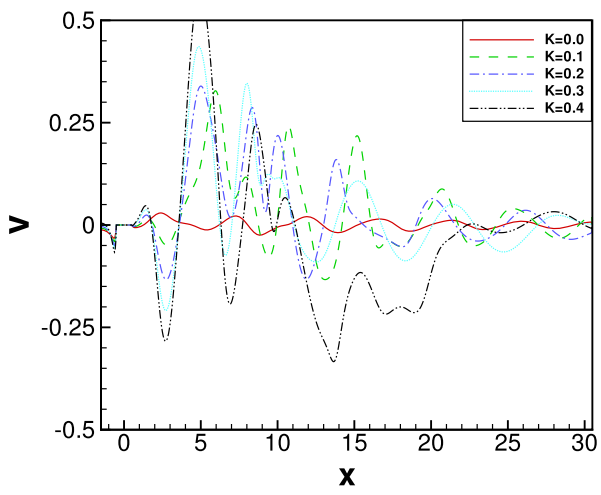

(b)

Fig. 9 Center-line velocity fluctuation for $\operatorname{Re}=100, s / d=2.0$ at different $K$ values $\mathbf{a} u$ along $y$-axis $\mathbf{b} v$ along $x$-axis 

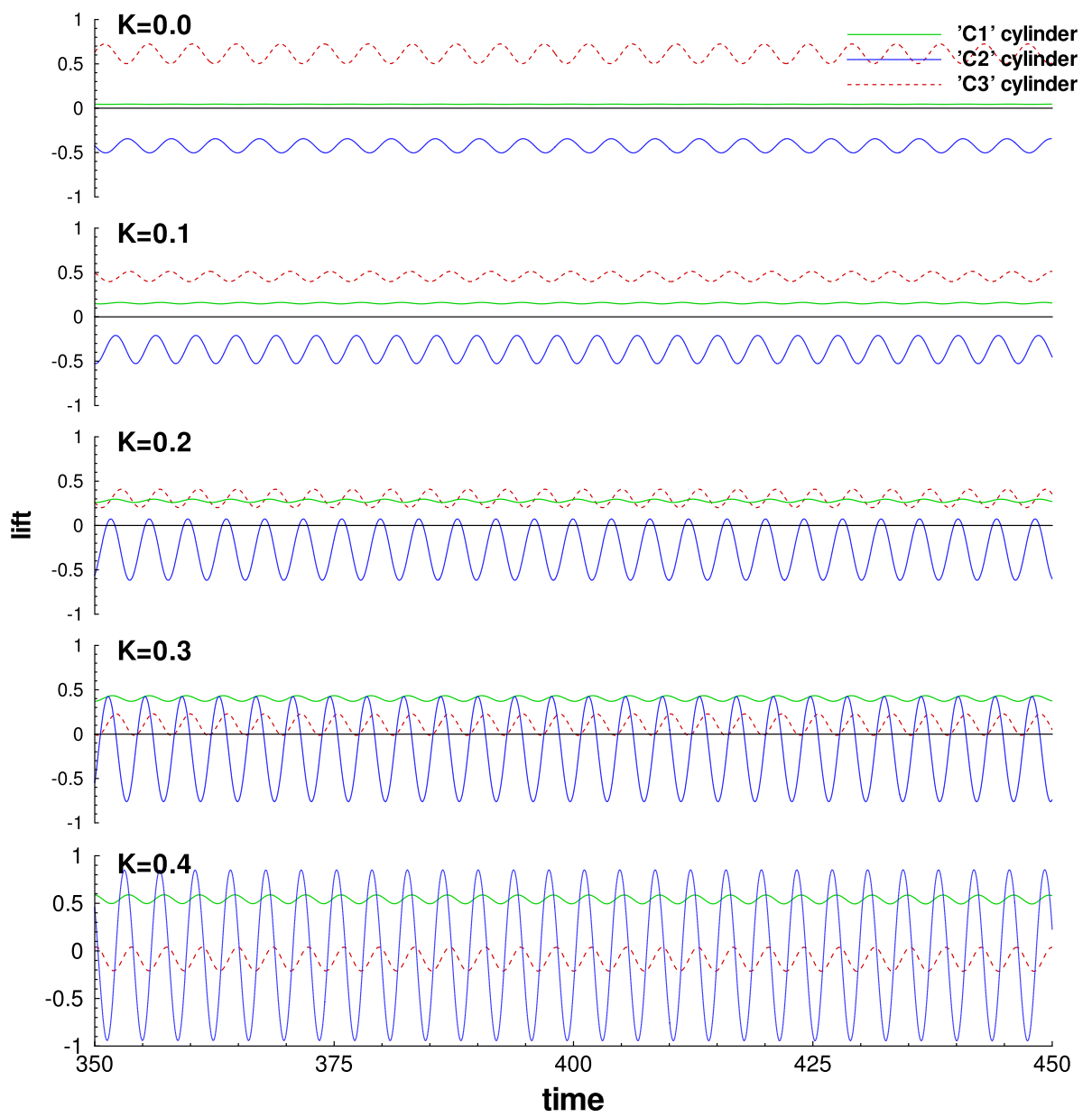

Fig. 10 Lift coefficient fluctuation with time for $s / d=2.0$

the wake centerline. Further, the vortex-shedding frequency is significantly decreased with increasing $K$ value. The phase difference between the vortices behind downstream cylinders also decreases with increasing $K$ value upto 0.3 , after that it starts to increase as mentioned in Table 3. The particles follow the similar path by the fluid flow to the downstream region, as shown by plotting the streakline patterns in Fig. 14. We further confirm the vortex-shedding process by plotting lift and drag coefficients with time in Figs. 15 and 16, respectively. In this case also, a phase shift between the vortex shedding of the downstream cylinders is seen from the signals of lift coefficients of the two cylinders ' $\mathrm{C} 2$ ' and ' $\mathrm{C} 3$ ' for all $K$ values. For $K=0.0$, the flow behind the upstream cylinder shows steady behavior while periodic flow maintains Von Karmann vortex street observed behind the downstream cylinders. With increasing $K$, the amplitude of fluctuation of lift and drag coefficients with single peak increases for ' $\mathrm{C} 2$ '. Also, the cylinder ' $\mathrm{C} 2$ ' experiences higher drag force with compare to ' $\mathrm{C} 3$ '. It is also to be noted that the magnitude of lift coefficient decreases gradually with increasing $K$ for 'C3'. Figures 17 and 18 present the axial profile of velocity fluctuations and phase diagram, respectively, for all $K$ values at $s / d=3.0$, which further confirm our observations. Here, we 


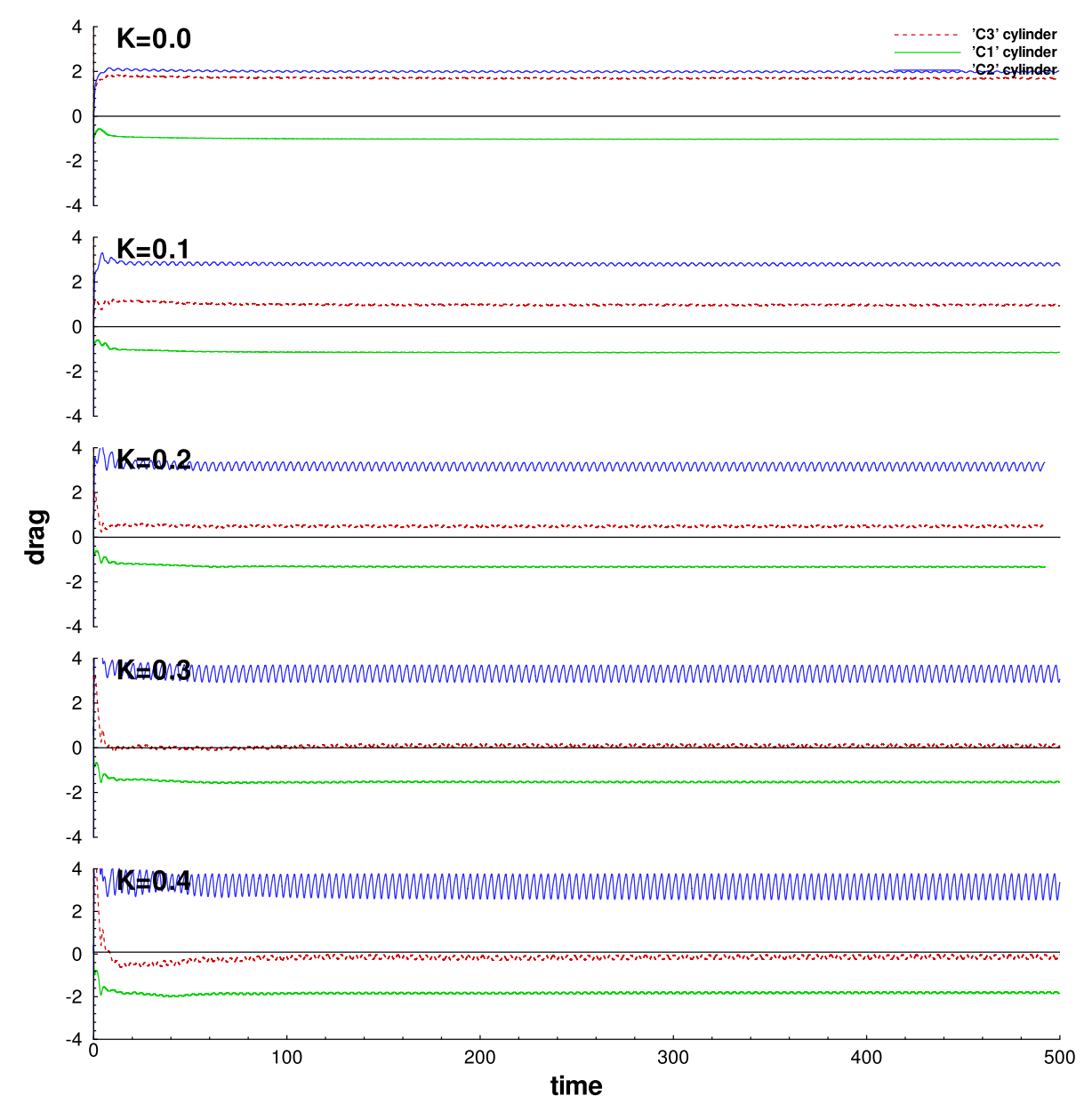

Fig. 11 Drag coefficient fluctuation with time for $s / d=2.0$

see that $u$ velocity shows similar behavior like previous $s / d$ ratios while $v$ velocity profile shows periodic nature with larger amplitude of oscillations.

\subsection{Structural bifurcation analysis of unsteady flow separation}

\subsubsection{Structural bifurcation}

The unsteady flow separation and mechanism of vortex shedding in the previous sections have been described mainly by qualitative observations. It is well known that the major source of instability in the topological structure of the 2-D incompressible fluid flows is the structural bifurcation, i.e., flow separation. The flow is unstable in the neighborhood of a bifurcation point. The bifurcation analysis is a very useful tool to explain the structural changes caused by unsteady flow separation in fluid flow around solid objects. In this section, we study the mechanism of structural bifurcation in the topological structure of the flow. 


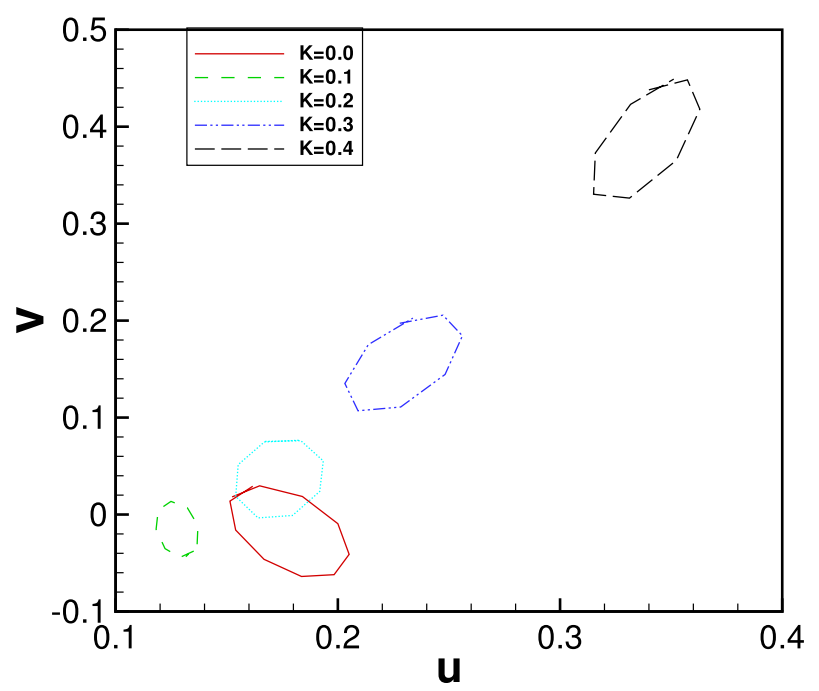

Fig. 12 Phase diagram for $\operatorname{Re}=100, s / d=2.0$ at different $K$ values

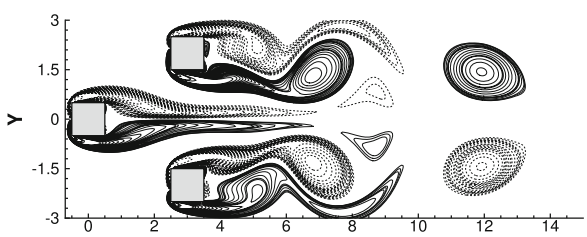

(a)

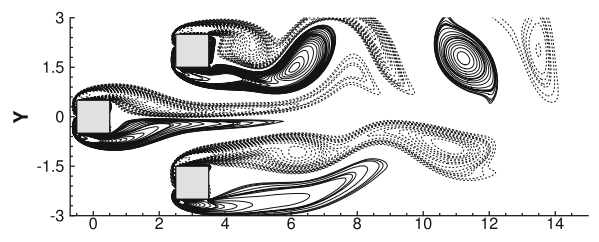

(b)

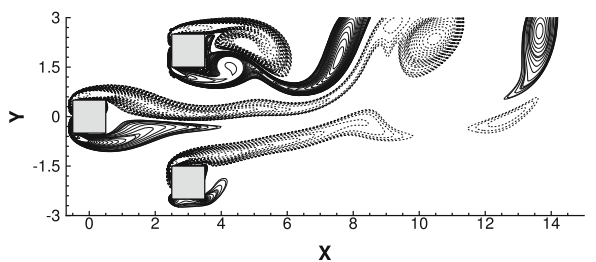

(d)

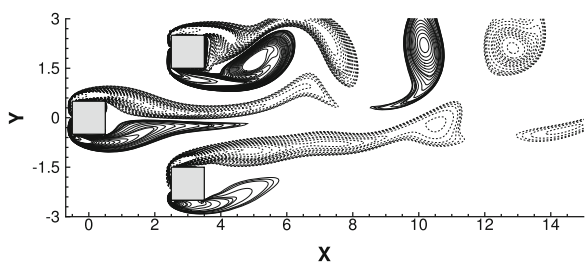

(c)

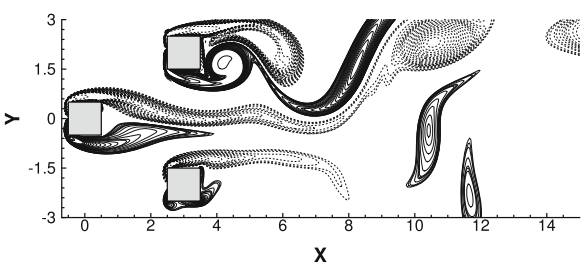

(e)

Fig. 13 Vorticity contours for fully developed flow at $\operatorname{Re}=100, s / d=3.0$, solid line contours represent positive vortex, dotted line contours represent negative vortex a $K=0.0$, b $K=0.1, \mathbf{c} K=0.2, \mathbf{d} K=0.3$, e $K=0.4$ 


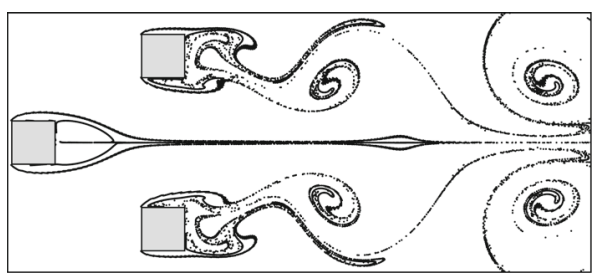

(a)

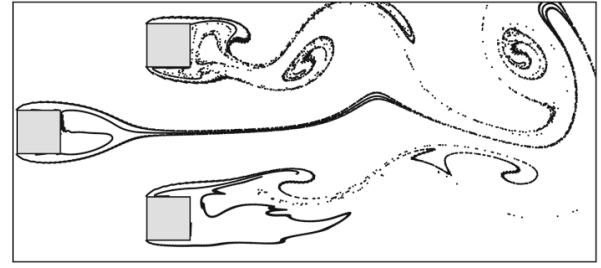

(b)

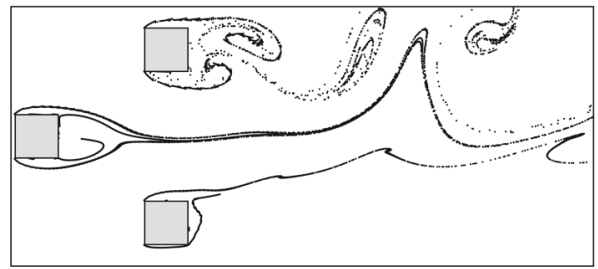

(d)

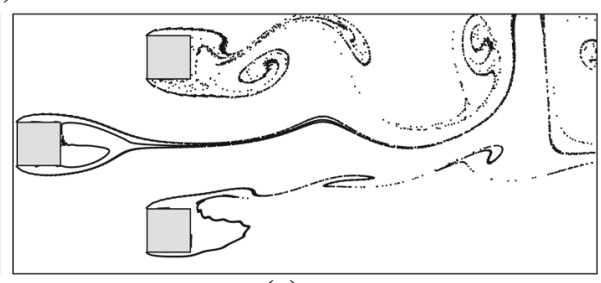

(c)

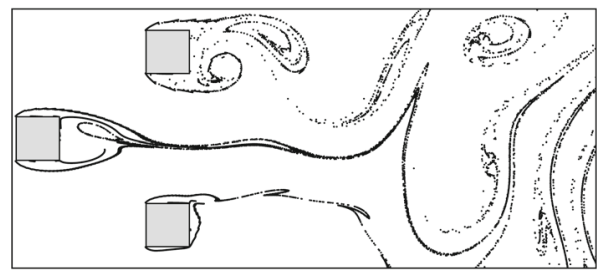

(e)

Fig. 14 Streakline patterns for $\operatorname{Re}=100, s / d=3.0$, a $K=0.0$, b $K=0.1$, c $K=0.2$, d $K=0.3$, e $K=0.4$

The unsteady flow separation from the surface of the cylinders and wake development described previously was mainly based on qualitative observations. Also, the unsteady flow separation from cylinder surface in case of shear flow past a single square cylinder using structural bifurcation theory has already been described in the author's recent work (Ray and Kumar 2017). The process of structural bifurcations seen many times in 2-D unsteady incompressible shear flow past three square cylinders in vee-shape arrangement. To the best of our knowledge, this type of study using bifurcation theory is not done yet to describe the flow phenomenon for this problem. We shall aim to do this in order to have a comprehensive study of the shear rate effect on the flow phenomenon using this theory. In a transient fluid flow, governed by partial differential equations, a bifurcation occurs when a small change made in the time parameter results a rapid change in the flow structure. Ghil et al. (2004, 2005) use this theory to study the boundary layer separation. The conditions for the flow separation at a point $P^{*}$ on the surface of the cylinder, at time $T^{*}$ is given as follows (15):

$$
\omega\left(P^{*}, T^{*}\right)=0, \frac{\partial \omega}{\partial \kappa}\left(P^{*}, T^{*}\right)=0, \frac{\partial^{2} \omega}{\partial \kappa^{2}}\left(P^{*}, T^{*}\right)>0, \frac{\partial \omega}{\partial t}\left(P^{*}, T^{*}\right)<0,
$$

where $\omega$ is the vorticity, $t$ is time and $\kappa$ is the tangential direction to the wall. It is assumed that if a structural bifurcation occurs at time $T^{*}$, the normal derivative of the velocity field $\frac{\partial U}{\partial \eta}$ (where $\eta$ is the direction outward normal to the wall) has a degenerate singular point $P^{*}$ on the solid surface with downward flow. In case of upward shear flow, last two inequalities in (15) will change the signs. To locate the bifurcation points, we have simultaneously solved $\mathrm{N}-\mathrm{S}$ equations with bifurcation conditions stated above in (15) to illustrate the separation 

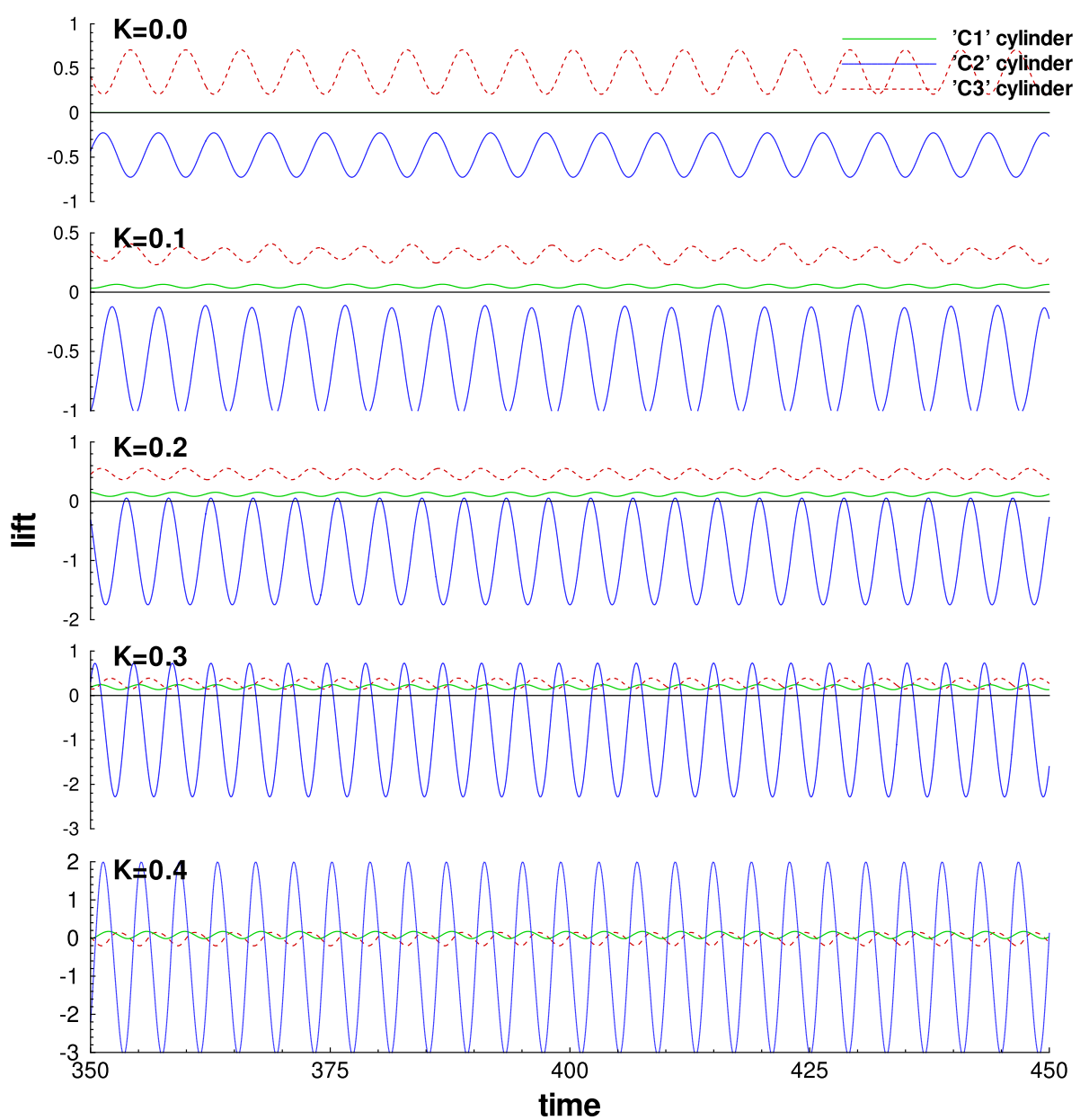

Fig. 15 Lift coefficient fluctuation with time for $s / d=3.0$

associated with the secondary and tertiary vortex phenomena. Note that the primary vortex for this problem develops due to the sharp corner of the square cylinders, that is why this vortex does not satisfy all the conditions stated in (15). So, in our study, we describe the structural bifurcation from the secondary vortex onward.

Figure 19 describes the occurrence of first and second structural bifurcation points corresponding to the pair of secondary vortices during flow separation from the surface of upstream cylinder ' $\mathrm{C} 1$ ' for $\mathrm{Re}=100, s / d=0.6$ at $K=0.0$. In Fig. 19a, we plot the vorticity $\omega$ (which is scaled down along vertical axis in order to make it comparable with the pressure) and pressure $p$ distribution along the surface of the cylinder ' $\mathrm{C} 1$ ' at time $t=0.879$. The variation of vorticity with time at the points $P_{1}^{*}(x, y)=(-0.34,0.5), P_{2}^{*}(x, y)=(-0.34,-0.5)$ is shown in Fig. 19b. Therefore, all the bifurcation conditions stated in (15) are satisfied. In this case, due to uniform flow, the first and second bifurcations occur at points $P_{1}^{* C 1}(x, y)=(-0.22,-0.5), P_{2}^{* C 2}(x, y)=(-0.22,0.5)$ at time $t=0.879$ as explained by Ray and Kumar (2017). When $t<T^{*}$, there is no singular point on the surface vorticity plot (Fig. 20d, e) in the neighborhood of $P_{1}^{* C 1}$ and $P_{2} * C 2$, respectively. The zoomed view 


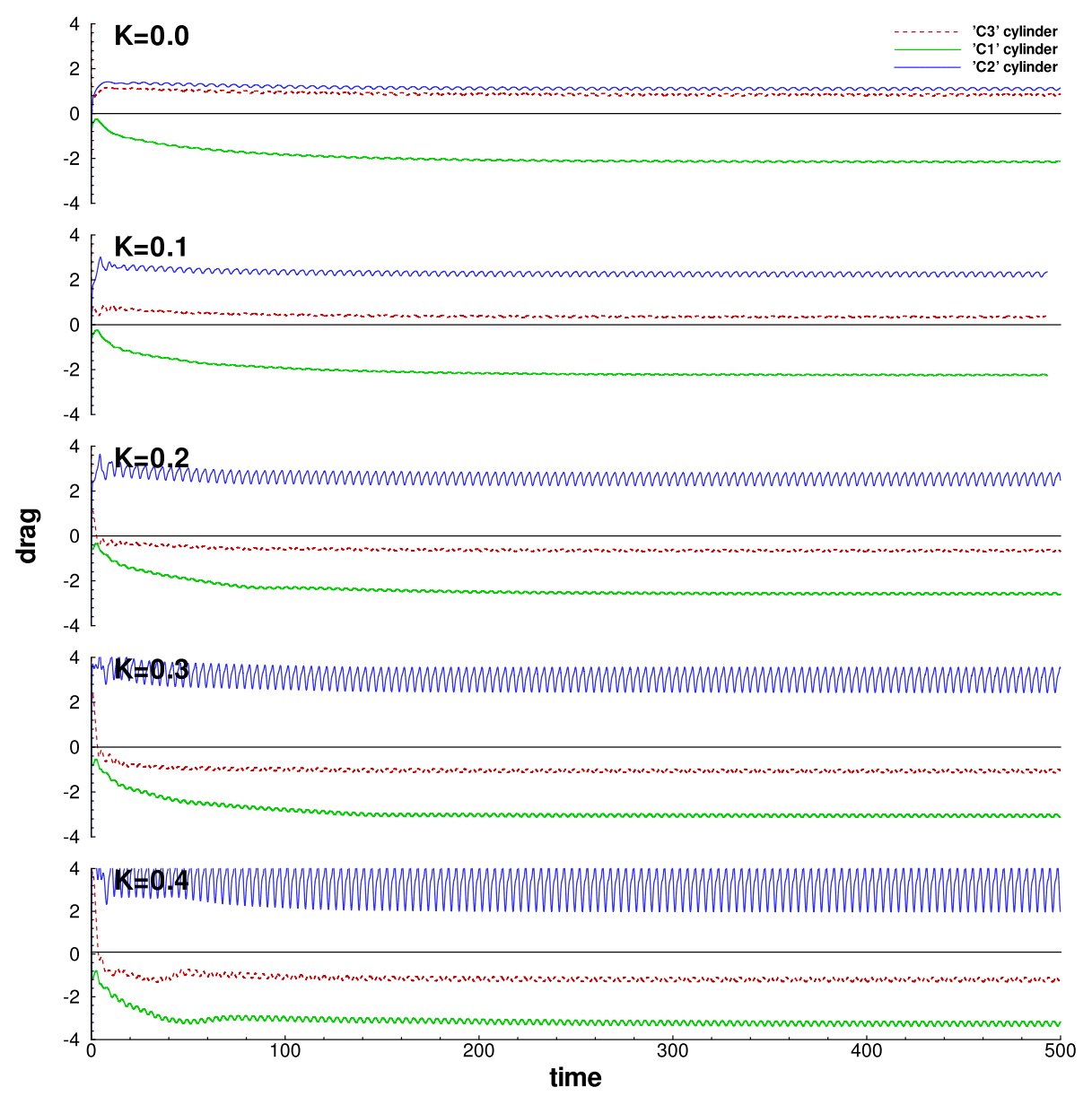

Fig. 16 Drag coefficient fluctuation with time for $s / d=3.0$

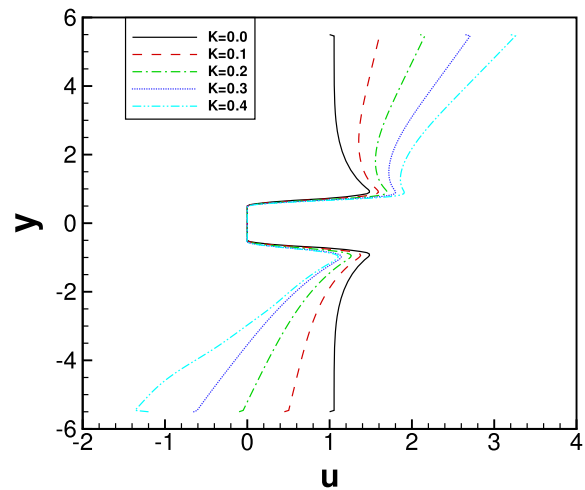

(a)

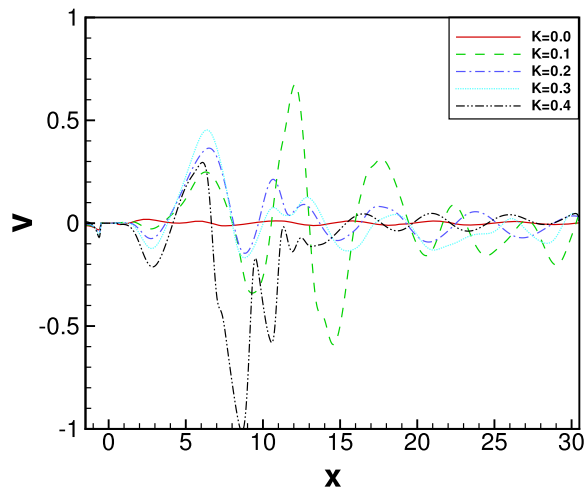

(b)

Fig. 17 Center-line velocity fluctuation for $\operatorname{Re}=100, s / d=3.0$ at different $K$ values $\mathbf{a} u$ along $y$-axis $\mathbf{b} v$ along $x$-axis 


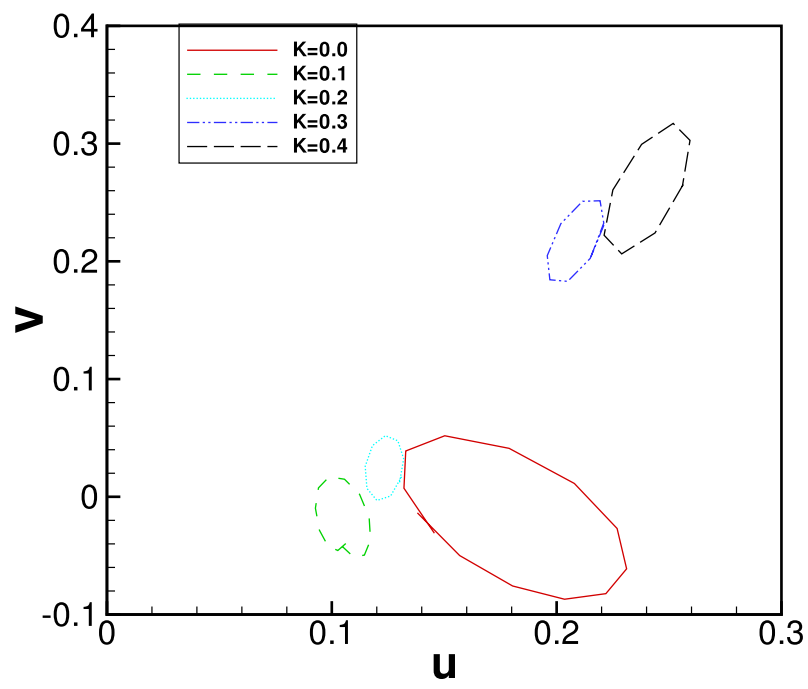

Fig. 18 Phase diagram for $\operatorname{Re}=100, s / d=3.0$ at different $K$ values

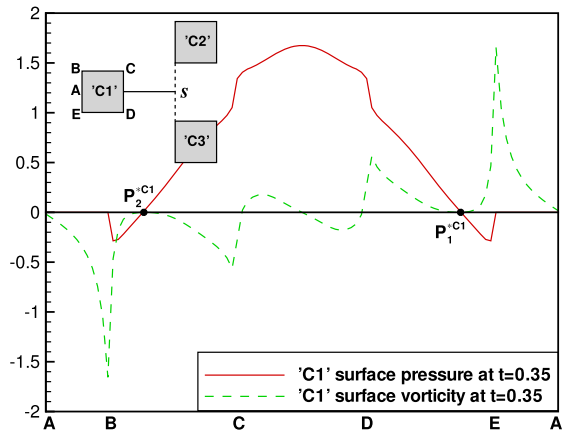

(a)

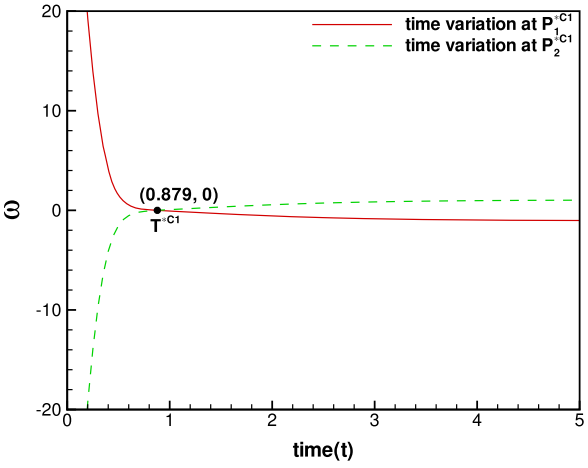

(b)

Fig. 19 a Vorticity and pressure distribution along the surface of the upstream square cylinder during the structural bifurcation at $t=0.879, \mathbf{b}$ time history of vorticity at points $P_{1}^{* C 1}(x, y)=(-0.22,-0.5)$, $P_{2}^{* C 2}(x, y)=(-0.22,0.5)$ on the surface of the upstream cylinder for $\operatorname{Re}=100$ and $s / d=0.6$ at $K=0.0$

of local structure of the stream lines near upper and lower sides of the cylinder is shown in Fig. 20b.

When $t=T^{*}$, there exists a pair of degenerate singular points in terms of $P_{1}^{* C 1}$ and $P_{2}^{* C 2}$ on the upper and lower sides of the cylinder, respectively. The zoomed plot of the local structure of the stream function near upper and lower sides of the cylinder at $t=T^{*}$ is shown in Fig. 20b.

When $t>T^{*}$, there exist two non-degenerate isolated singular points, $P_{1}(x, y)=$ $(-0.38,0.5), P_{2}(x, y)=(-0.18,0.5)$ on the upper side and $P_{1}(x, y)=(-0.38,-0.5)$, $P_{2}(x, y)=(-0.18,-0.5)$ on the lower side of the upstream cylinder, on the surface vorticity plot (Fig. 20d, e) at $t=1.2$, respectively. Figure 20c shows the zoomed view of local structure of stream function near upper and lower sides of the cylinder. 


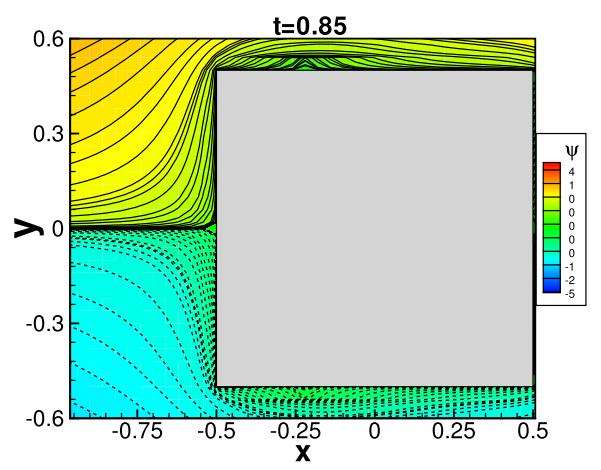

(a)

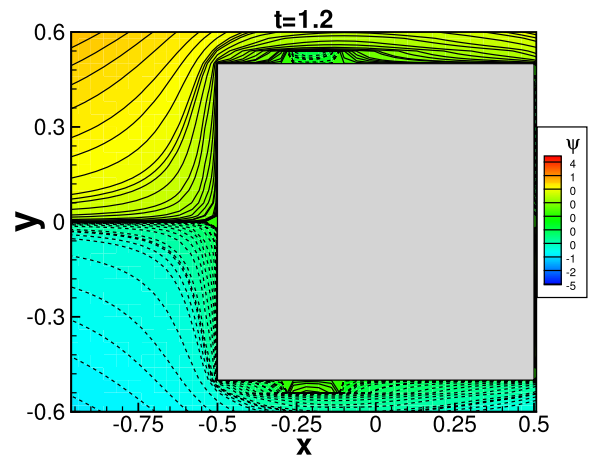

(c)

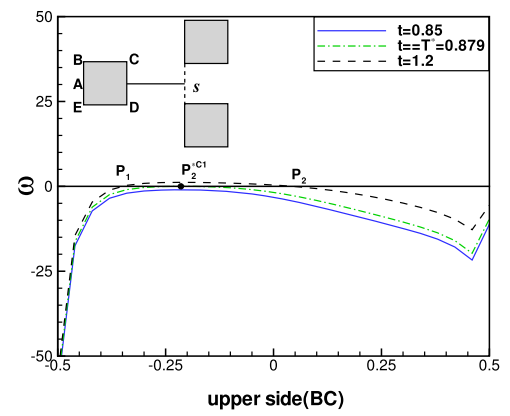

(d)

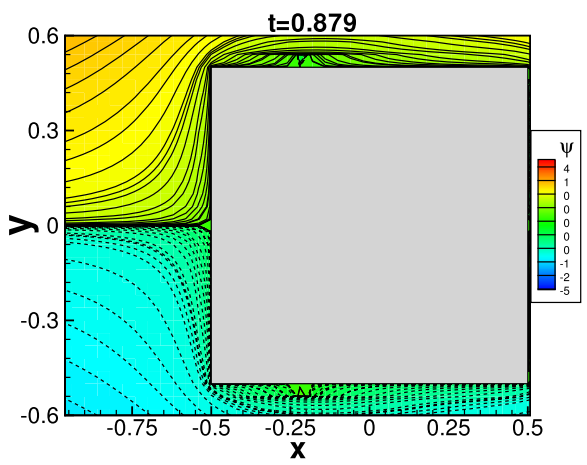

(b)

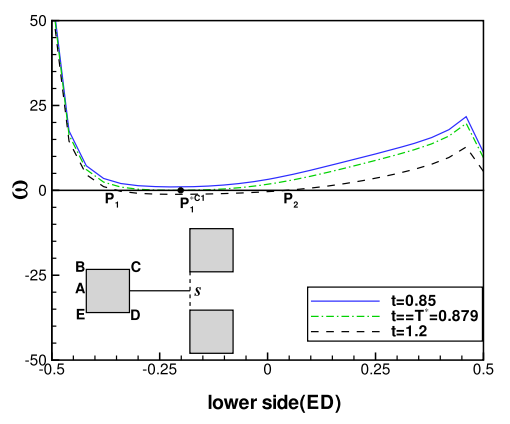

(e)

Fig. 20 For $\operatorname{Re}=100, s / d=0.6$ and $K=0.0$, zoomed view of streamlines in the region $[-0.75,0.5] \times[-0.6$, $0.6]$ of upstream cylinder at $\mathbf{a} t=0.85, \mathbf{b} t=0.879$, $\mathbf{c} t=1.2$, d vorticity profile along the upper side of the upstream cylinder, e vorticity profile along the lower side of the upstream cylinder

Now, we describe the process of structural bifurcation for $K=0.1$. We already have seen that flow is asymmetric around wake centerline for a non-zero value of $K$. Owing to asymmetric nature of the flow, the first structural bifurcation occurs at point $P_{1}^{* C 1}=$ $(-0.22,-0.5)$ at time $t=0.726$, second bifurcation occurs at point $P_{2}^{* C 2}=(-0.22,0.5)$ and at time $t=1.135$ on lower and upper sides of the upstream cylinder, respectively (Fig. 21). 


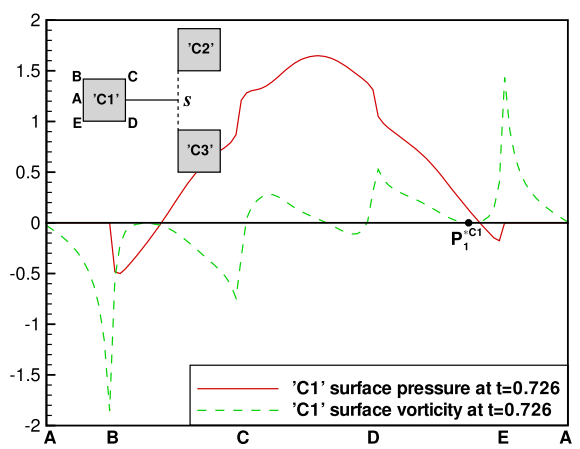

(a)

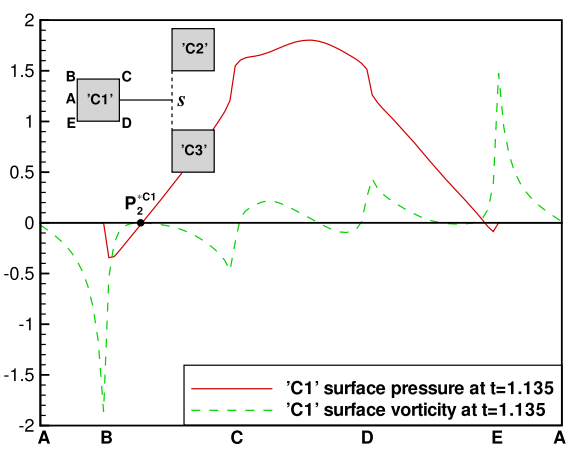

(b)

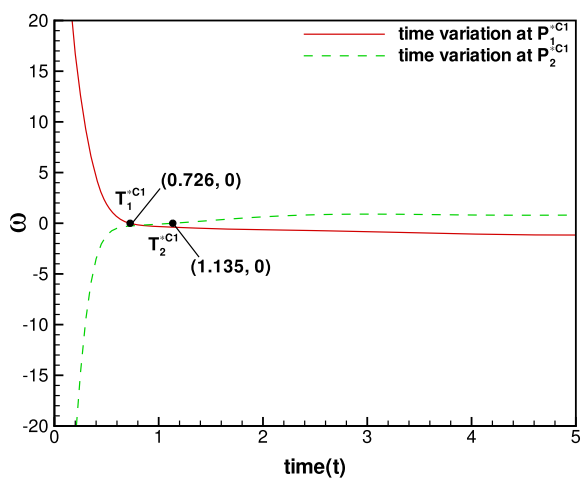

(c)

Fig. 21 Vorticity and pressure distribution along the surface of the upstream cylinder at $\mathbf{a} t=0.726$, b $t=1.135$ and $\mathbf{c}$ time history of vorticity at points $P_{1}^{* C 1}(x, y)=(-0.22,-0.5), P_{2}^{* C 2}(x, y)=(-0.22,0.5)$ on the surface of the upstream cylinder for $\operatorname{Re}=100, s / d=0.6$ at $K=0.1$

Further, the occurrence of structural bifurcations for $s / d=1.0$ at $K=0.0$ from the surface of upstream cylinder is shown in Fig. 22. As cylinders ' $\mathrm{C} 2$ ' and ' $\mathrm{C} 3$ ' also undergo the process of structural bifurcation in its topological structure, we can see that the first structural bifurcation on downstream cylinders ' $\mathrm{C} 2$ ' and ' $\mathrm{C} 3$ ' occurs at same time for $K=0.0$, owing to symmetric nature of flow around wake centerline. Figure 23 shows the occurrence of first structural bifurcation on downstream cylinders. The surface vorticity (which is scaled down along vertical axis in order to make it comparable with the pressure) and pressure distribution on both downstream cylinders are plotted at time $t=3.634$; see Fig. 23a, b. The time variation of vorticity at points $P_{1}^{* C 2}=(2.66,1.3), P_{1}^{* C 3}=(2.66,-1.3)$ is shown in Fig. 23c. From the figures, it is clear that vorticity reaches zero as a local minima at point $P_{1}^{* C 2}$ and further decreases with time, as a local maxima at point $P_{1}^{* C 3}$ and further increases with time, respectively. This implies that first structural bifurcation on downstream cylinders occurs at points $P_{1}^{* C 2}=(2.66,1.3), P_{1}^{* C 3}=(2.66,-1.3)$ at time $t=3.634$.

Finally, the details of bifurcation points with time for all parameter values are shown in Tables 4, 5. We can see that, maximum two structural bifurcations occur on all three cylinder 


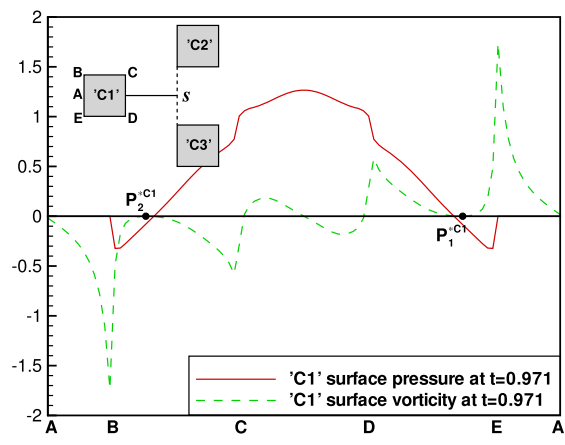

(a)

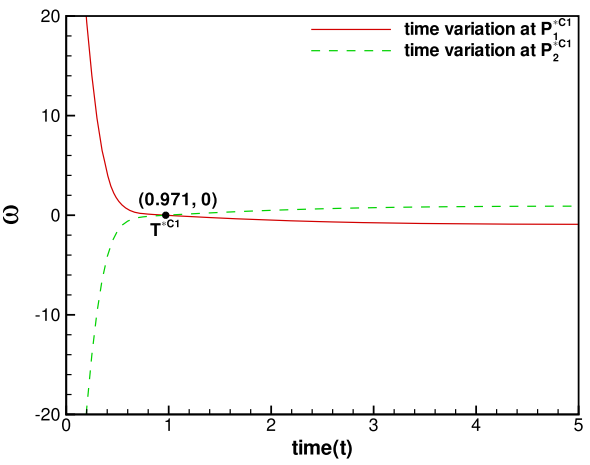

(b)

Fig. 22 a Vorticity and pressure distribution along the surface of the upstream cylinder during the structural bifurcation at $t=0.971, \mathbf{b}$ time history of vorticity at points $P_{1}^{* C 1}(x, y)=(-0.22,-0.5), P_{2}^{* C 1}(x, y)=$ $(-0.22,0.5)$ on the surface of the upstream cylinder for $\operatorname{Re}=100$ and $s / d=1.0$ at $K=0.0$

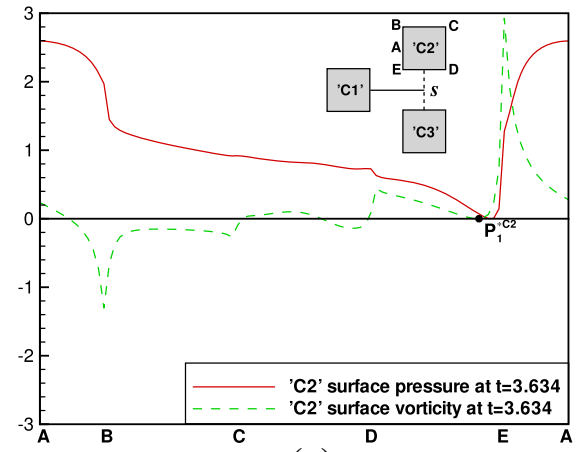

(a)

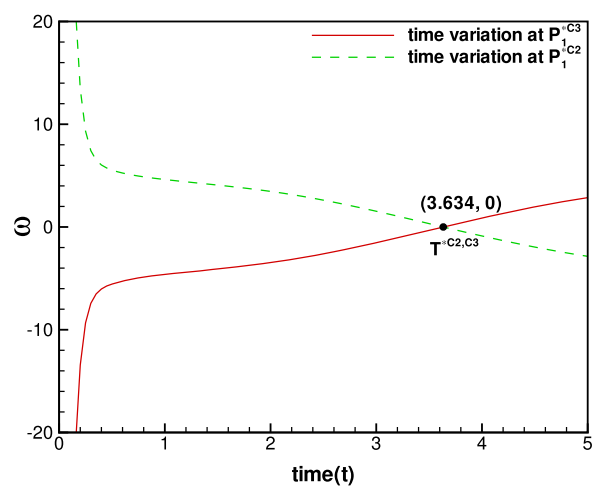

(c)

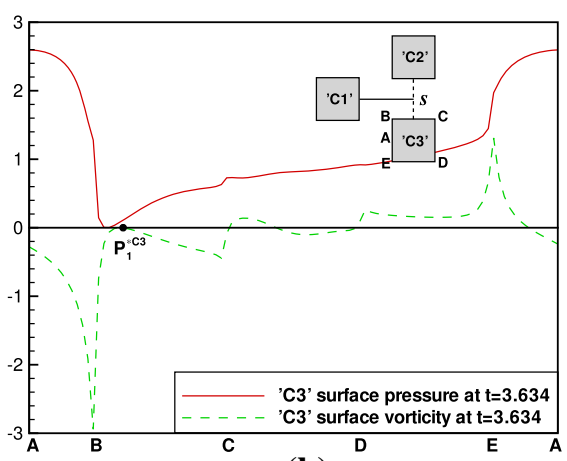

(b)

Fig. 23 Vorticity and pressure distribution along the surface of the cylinders a ' $\mathrm{C} 2$ ' and b ' $\mathrm{C} 3$ ' during the structural bifurcation at $t=3.634$, $\mathbf{c}$ time history of vorticity at points $P_{1}^{* C 2}(x, y)=(2.70,0.5)$ on the surface of ' $\mathrm{C} 2$ ', $P_{1}^{* C 3}(x, y)=(2.70,-0.5)$ on the surface of ' $\mathrm{C} 3$ ' for $\operatorname{Re}=100$ and $s / d=1.0$ at $K=0.0$ 
for all parameter values due to proximity effect of downstream cylinders 'C2' and 'C3'; see Tables 4,5 . It is also worthy to mention that the bifurcations occur symmetrically on the cylinder surface for $K=0.0$ in all the cases. The first structural bifurcation on the upstream cylinder always occurs from the upper side for all $s / d$ ratios, and the time of occurrence is decreased with increasing $K$ value (Table 4). On the other hand, the time of the second bifurcation occurs on lower side increases with increasing $K$ values. The first two bifurcations on the upstream cylinder occur in a very short duration after the initial start of the flow. Each downstream cylinder undergoes only two structural bifurcations in its topological structure. For $s / d=0.6$, the only bifurcation occurs from upper and lower side of the cylinders ' $\mathrm{C} 2$ ' and 'C3', respectively, at $K=0.0$. The square cylinder ' $\mathrm{C} 3$ ' has no structural bifurcation for $K>0.1$ for $s / d=0.6$; see Table 5 . Overall, the second structural bifurcation does not occur on cylinder ' $\mathrm{C} 3$ ' for larger values of $K$ for all $s / d$ ratios, due to the high relative velocity of fluid flowing in the upper region of wake centerline than the lower region (Table 5).

\subsection{Existence of saddle points}

In this section, the flow topology in terms of center (i.e., point of zero velocity) of vortices and saddle (i.e., the point of intersection of two streamlines) points has been described for this problem (Perry et al. 1982; Eton 1987). The existence of saddle points behind the cylinder for the shear flow past square cylinder has been described in our recent work Ray and Kumar (2017). For the case of shear flow past three square cylinders in vee-shape arrangement, saddle points exist in between the upstream and downstream cylinders as well as behind the downstream cylinders. The vortex centers and saddle points are detected for initial flow shown in Fig. 24 for $\operatorname{Re}=100, s / d=0.6,3.0$ for $K=0.0,0.2$ and 0.4. The occurrence of these critical points (center and saddle) considerably differs with $s / d$ ratios and $K$ values. Figure 24a shows the existence of saddle points and a pair of center of vortices behind each cylinder for $s / d=0.6$ at $K=0.0$. In this case, a pair of counter-rotating vortices with centers $C_{1}=(0.68,0.34), C_{2}=(0.68,-0.34)$ of similar size with a common saddle point $S_{1}=(0.81,0)$ exist behind the upstream cylinder. On the other hand, the vortices with centers $C_{3}=(3.62,1.12), C_{4}=(3.85,0.56)$ with a saddle point $S_{2}=(3.96,0.81)$; $C_{5}=(3.85,-0.56), C_{6}=(3.62,-1.12)$ with a saddle point $S_{3}=(3.96,-0.81)$ of different sizes exist behind the downstream cylinders 'C2', 'C3', respectively. For $K=0.2$, the vortices with centers $C_{1}=(0.7144,0.2725), C_{2}=(0.6112,-0.3206)$, a saddle point $S_{1}=(0.8389,-0.0481)$ behind upstream cylinder develop asymmetrically around wake centerline. The saddle points behind downstream cylinders also exist in a similar way; see Fig. 24b. Further increase in $K$ value increases the wake fluctuation, results in a more complex flow structure behind the cylinders. In Fig. 24c, we show the position of the center of vortices and saddle points for $K=0.4$. In this case, a pair of vortices with centers $C_{1}, C_{2}$ behind the upstream cylinder (for $K=0.0$ ) transforms into a single vortex with center $C_{1}=(0.7913,0.1956)$ with a saddle point $S_{1}=(0.8543,-0.2636)$ for $K=0.4$. One thing is also noticed that the vortices with centers $C_{3}, C_{4}$ on the surface of the downstream cylinder ' $\mathrm{C} 3$ ' have no saddle point. Owing to the high shear, a big re-circulation zone is created around the cylinder ' $\mathrm{C} 3$ ' with a saddle point $S_{3}=(1.1215,-1.7591)$ (Fig. 24c). The saddle points move towards (-)ve $y$-direction with increasing $K$ value. A similar type of mechanism applied to locate the saddle points for $s / d=3.0$ is also shown in Fig. 24. 


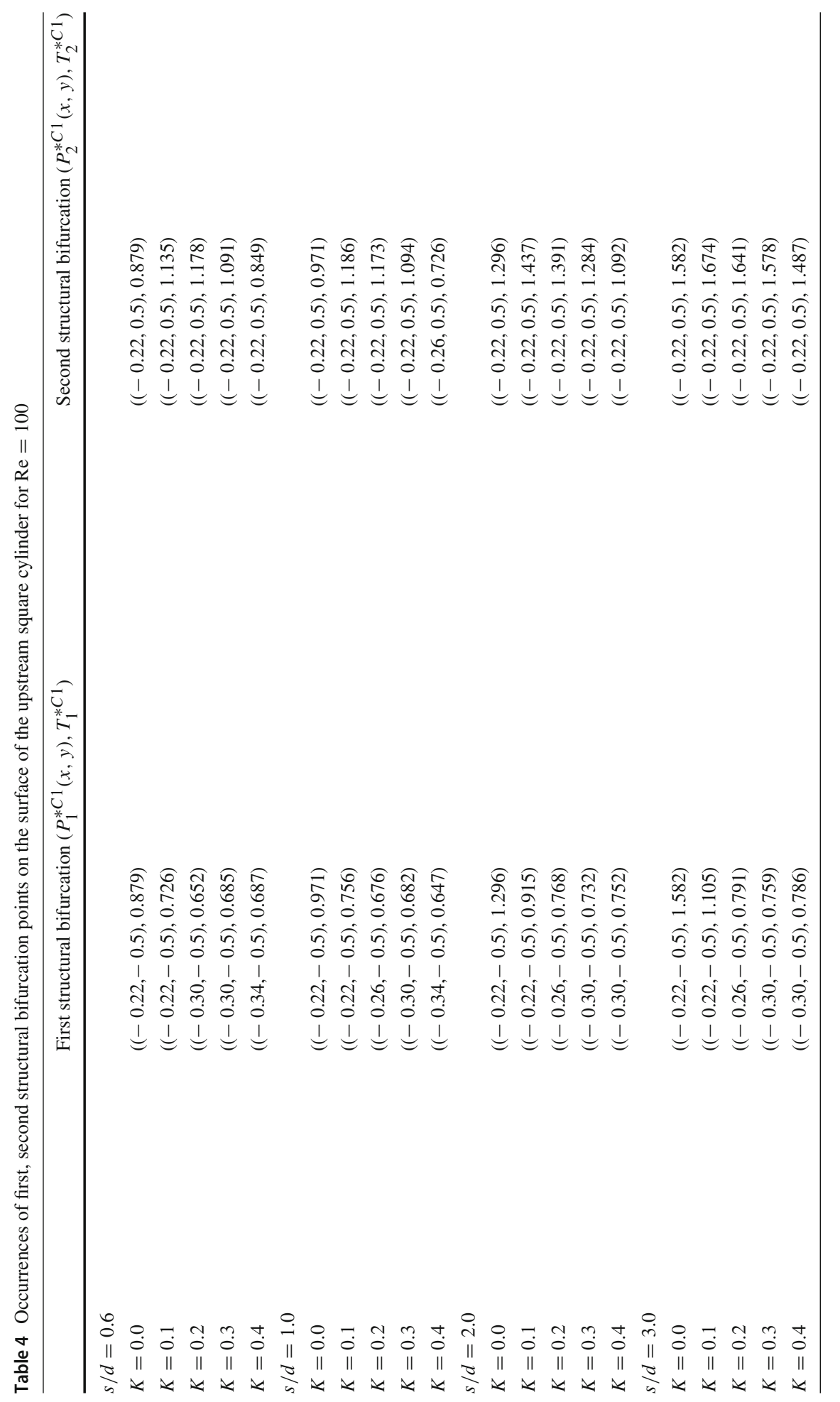




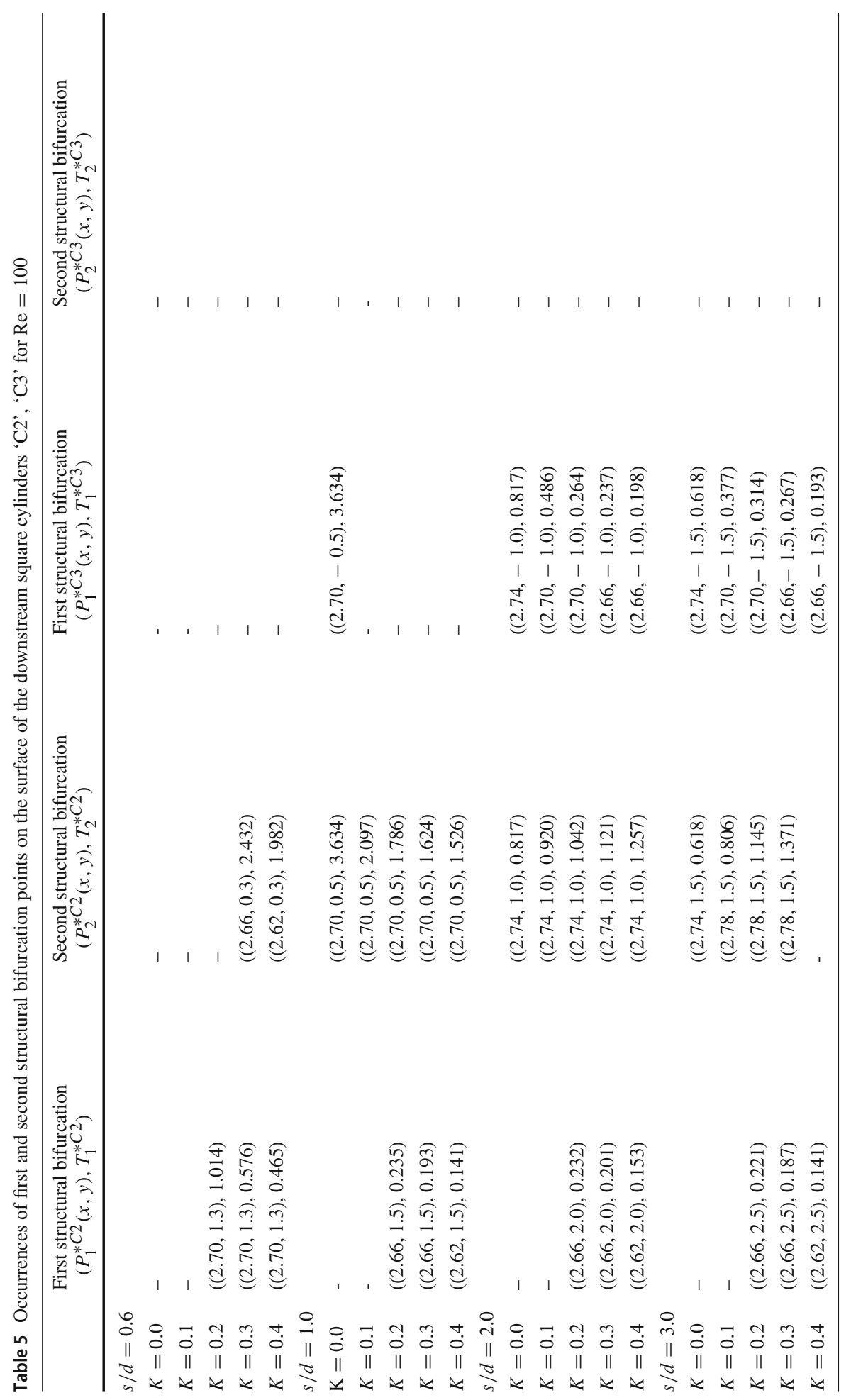

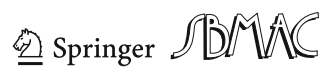




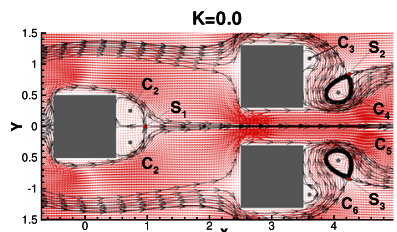

(a) $\mathrm{s} / \mathrm{d}=0.6$

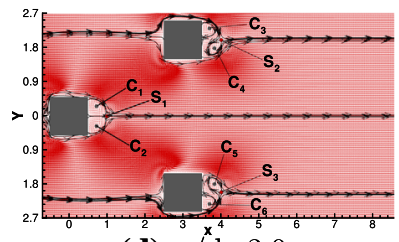

(d) $\mathrm{s} / \mathrm{d}=3.0$

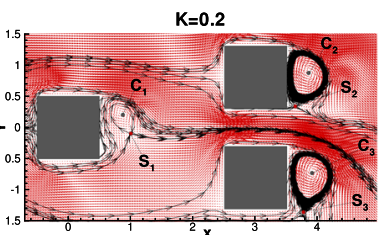

(b) $\mathrm{s} / \mathrm{d}=0.6$

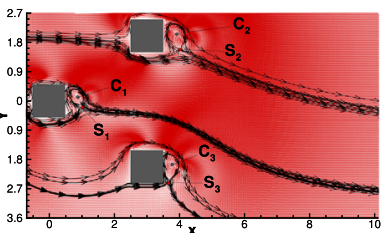

(e) $s / d=3.0$

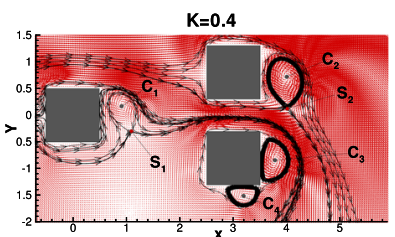

(c) $\mathrm{s} / \mathrm{d}=0.6$

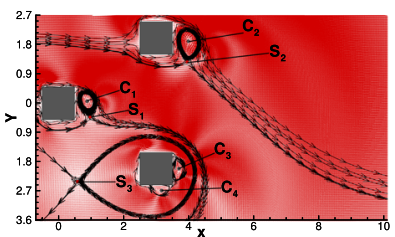

(f) $\mathrm{s}^{4} \mathrm{~d} d=3^{6} .0$

Fig. 24 Topological flow structures: velocity vector plots, streamline patterns showing center $\left(C_{1}, C_{2}, C_{3}, C_{4}, C_{5}, C_{6}\right)$ of vortices and saddle $\left(S_{1}, S_{2}, S_{3}\right)$ points for $\operatorname{Re}=100, s / d=0.6,3.0$ and $K=0.0,0.2,0.4$ at $t=1.0$

\section{Conclusion}

In this study, the unsteady flow separation for 2-D incompressible shear flow past three identical square cylinders arranged in vee shape is discussed for $\operatorname{Re}=100, s / d=0.6-3.0$ and $K=0.0$ to 0.4 . Topological aspect-based structural bifurcation analysis is used to find the exact location and time of occurrence of bifurcation points associated with secondary and tertiary vortices. Our study reveals that for $K=0.0$, i.e., for no shear flow case, two bifurcation points corresponding to a pair of secondary vortices appear symmetrically on upper and lower sides of the upstream cylinder for all $s / d$ ratios. We also observe that flow does not separate from the top and bottom surface of the downstream cylinder ' $\mathrm{C} 3$ ' for $s / d=0.6$ for any $K$ values considered here. For the upstream cylinder, the first bifurcation always occurs from the lower side for non-zero values of $K$. This is because the flow from the lower side of the cylinder has low relative velocity. Upon increasing the shear rate, the position of bifurcation point on top side becomes fixed, whereas at the bottom side bifurcation point gradually shifts upward. The values of $s / d$ and $K$ significantly affect the location and time of appearance of bifurcation points. It is worth mentioning that the downstream cylinders undergo less structural bifurcations than the upstream cylinder. The existence of saddle points are also reported for initial flow. It is observed that, for $s / d=0.6,3.0$ at $K=0.0$, one saddle point occurs behind each cylinder symmetrically about the centerline. With increasing $K$, the saddle point moves downward direction due to the asymmetric nature of the flow.

In this process, we have also visualized the vortex-shedding phenomenon caused by unsteady flow separation. The fully developed flow is studied in terms of vorticity contours, streakline patterns, center-line velocity fluctuation and phase diagrams for all parameter values considered here. Some interesting flow phenomena are observed for high shear parameter values. It is observed that for $s / d=2.0,3.0$, a definite phase relationship exist between shedding of vortices behind the downstream cylinders ' $\mathrm{C} 2$ ' and ' $\mathrm{C} 3$ '. The phase difference decreases continuously for $s / d=2.0$ with increasing $K$ values considered here. However, it decreases upto $K=0.3$ for $s / d=3.0$ and then starts to increase. Also, the phase difference 
has smaller values for $s / d=2.0$ in comparison to $s / d=3.0$ for non-zero values of $K$. Our observations also show that the vortex-shedding phenomenon in between and behind the cylinders significantly depends on $K$ and $s / d$ ratios. The differences in the size and strength of the alternatively shedding vortices become more prominent with increasing $K$ values.

Acknowledgements The authors would like to acknowledge SERB (DST), Govt. of India, for providing the financial support under project (no.: SERB/F/7046/2013-2014 dated 12.02.2014).

\section{Appendix 1}

The expressions for the finite difference operators appearing in the above equations are as follows:

$$
\begin{aligned}
\delta_{x} \phi_{i, j} & =\frac{\phi_{i+1, j}-\phi_{i-1, j}}{2 h}, \\
\delta_{y} \phi_{i, j} & =\frac{\phi_{i, j+1}-\phi_{i, j-1}}{2 h}, \\
\delta_{x}^{2} \phi_{i, j} & =\frac{\phi_{i+1, j}-2 \phi_{i, j}+\phi_{i-1, j}}{h^{2}}, \\
\delta_{y}^{2} \phi_{i, j} & =\frac{\phi_{i, j+1}-2 \phi_{i, j}+\phi_{i, j-1}}{h^{2}}, \\
\delta_{x} \delta_{y} \phi_{i, j} & =\frac{\phi_{i+1, j+1}-\phi_{i+1, j-1}-\phi_{i-1, j+1}+\phi_{i-1, j-1}}{4 h^{2}}, \\
\delta_{x}^{2} \delta_{y} \phi_{i, j} & =\frac{\phi_{i+1, j+1}-\phi_{i+1, j-1}-2\left(\phi_{i, j+1}-\phi_{i, j-1}\right)+\phi_{i-1, j+1}-\phi_{i-1, j-1}}{2 h^{3}}, \\
\delta_{x} \delta_{y}^{2} \phi_{i, j} & =\frac{\phi_{i+1, j+1}-\phi_{i-1, j+1}-2\left(\phi_{i+1, j}-\phi_{i-1, j}\right)+\phi_{i+1, j-1}-\phi_{i-1, j-1}}{2 h^{3}}, \\
\delta_{x}^{2} \delta_{y}^{2} \phi_{i, j} & =\frac{\phi_{i+1, j+1}+\phi_{i-1, j+1}-2 \phi_{i, j+1}-2 \phi_{i+1, j}+4 \phi_{i, j}-2 \phi_{i, j-1}-2 \phi_{i-1, j}+\phi_{i+1, j-1}+\phi_{i-1, j-1}}{h^{4}} .
\end{aligned}
$$

\section{References}

Bakker PG (1989) Bifurcation in flow patterns. PhD thesis, Technical University of Delft, Netherlands

Bao Y, Zhou D, Huang C (2010) Numerical simulation of flow over three circular cylinders in equilateral arrangements at low Reynolds number by a second-order characteristic-based split finite element method. Comput Fluids 39:882-899

Chatterjee D, Biswas G (2015) Dynamic behavior of flow around rows of square cylinders kept in staggered arrangement. J Wind Eng Ind Aerodyn 136:1-11

Chatterjee D, Gupta SK (2015) Convective transport around rows of square cylinders arranged in a staggered fashion at moderate Reynolds number. Numer Heat Transf Part A 68:388-410

Chatterjee D, Biswas G, Amiroudine S (2009) Numerical investigation of forced convection heat transfer in unsteady flow past a row of square cylinders. Int J Heat Fluid Flow 30(6):1114-1128

Cheng M, Whyte DS, Lou J (2007) Numerical simulation of flow around a square cylinder in uniform-shear flow. J Fluids Struct 23:207-226

Eton BE (1987) Analysis of laminar vortex shedding behind a circular cylinder by computer-aided flow visualization. J Fluid Mech 180:117-145

Ghil M, Liu JG, Wang C, Wang S (2004) Boundary-layer separation and adverse pressure gradient for 2-D viscous incompressible flow. Phys D 197:149-173

Ghil M, Ma T, Wang S (2005) Structural bifurcation of 2-D nondivergent flows with Dirichlet boundary conditions applications to boundary-layer separation. SIAM J Appl Maths 65:1576-1596 
Gu Z, Sun T (2001) Classifications of flow pattern on three circular cylinders in equilateral-triangular arrangements. J Wind Eng Ind Aerodyn 89:553-568

Gurcan F, Deliceoglu A, Bakker PG (2005) Streamline topologies near a non-simple degenerate critical point close to a stationary wall using normal forms. J Fluid Mech 539:299-311

Hawang Robert R, Sue YC (1997) Numerical simulation of shear effect on vortex shedding behind a square cylinder. Int J Numer Methods Fluids 25:1409-1420

Igarashi T (1981) Characteristics of the flow around two circular cylinders arranged in tandem. Bull ASME 24:188

Inoue O, Mori M, Hatakeyama N (2006) Aeolian tones radiated from flow past two square cylinders in tandem. Phys Fluids 18:046101

Kalita JC, Sen S (2013) Unsteady separation leading to secondary and tertiary vortex dynamics: the sub- $\alpha$ and sub- $\beta$-phenomena. J Fluid Mech 730:19-51

Kalita JC, Dalal DC, Dass AK (2002) A class of higher order compact schemes for the unsteady twodimensional convection-diffusion equation with variable convection coefficients. Int J Numer Meth Fluids 38:1111-1131

Kang S (2006) Uniform-shear flow over a circular cylinder at low Reynolds numbers. J Fluids Struct 22:541555

Kiya M, Tamura H, Arie M (1980) Vortex shedding from a circular cylinder in moderate reynolds number shear flow. J Fluid Mech 141:721-735

Kumar A, Ray RK (2015) Numerical study of shear flow past a square cylinder at Reynolds numbers 100, 200. Procedia Eng 127:102-109

Kumar A, Ray RK (2017) A numerical simulation of shear flow past two equal sized square cylinders arranged in parallel at $\mathrm{Re}=500$. AIP Conf Proc 1863(1):490003

Kumar A, Ray RK (2018) Numerical simulation of flow around square cylinder with an inlet shear in a closed channel. Springer, Singapore

Kumar A, Ray RK (2016) Higher order compact numerical simulation of shear flow past inclined square cylinder. In: Advanced computing and communication technologies, pp 305-313

Lankadasu A, Vengadesan S (2007) Interference effect of two equal-sized square cylinders in tandem arrangement: with planar shear flow. Int J Numer Meth Fluids 57:1005-1021

Lankadasu A, Vengadesan S (2008) Onset of vortex shedding in planar shear flow past a square cylinder. Int J Heat Fluid Flow 29:1054-1059

Lankadasu A, Vengadesan S (2010) Shear effect on square cylinder wake transition characteristics. Int J Numer Meth Fluids 67:1112-1134

Li H, Sumner D (2009) Vortex shedding from two finite circular cylinders in a staggered configuration. J Fluids Struct 25:479-505

Luo SC, Chew YT, Ng YT (2003) Characteristics of square cylinder wake transition flows. Phys Fluids 15(9):2549-2559

Ma T, Wang S (2002) Topology of 2-D incompressible flows and applications to geophysical fluid dynamics. Matematicas 96:447-459

Mittal S, Kumar V, Raghuvanshi A (1997) Unsteady incompressible flows past two cylinders in tandem and staggered arrangements. Int J Numer Meth Fluids 25:1315-1344

Mittal HVR, Al-Mdallal QM, Ray RK (2017) Locked-on vortex shedding modes from a rotationally oscillating circular cylinder. Ocean Eng 146:324-338

Moussaoui MA, Mezrhab A, Naji H (2011) A computation of flow and heat transfer past three heated cylinders in a vee shape by a double distribution MRT thermal lattice Boltzmann model. Int J Therm Sci 50:15321542

Ozgoren M (2013) Flow structures around an equilateral triangle arrangement of three spheres. Int J Multiph Flow 53:54-64

Perry AE, Chong MS, Lim TT (1982) The vortex shedding process behind two-dimensional bluff bodies. J Fluid Mech 116:77-90

Ray RK, Kalita JC (2010) A transformation-free HOC scheme for incompressible viscous flows on nonuniform polar grids. Int J Numer Meth Fluids 62(6):683-708

Ray RK, Kumar A (2017) Numerical study of shear rate effect on unsteady flow separation from the surface of the square cylinder using structural bifurcation analysis. Phys Fluids 29:083604

Saha AK, Biswas G, Muralidhar K (1999) Influence of inlet shear on structure of wake behind a square cylinder. J Eng Mech 125(3):359-363

Saha AK, Muralidhar K, Biswas G (2000) Vortex structures and kinetic energy budget in two-dimensional flow past a square cylinder. Comput Fluids 29(6):669-694

Saha AK, Biswas G, Muralidhar K (2001) Two-dimensional study of the turbulent wake behind a square cylinder subject to uniform shear. J Fluids Eng 123(3):595-603 
Zdravkovich MM (1977) Review of flow interference between two circular cylinders in various arrangements. J Fluids Eng (ASME) 99:618-633

Zdravkovich MM (1987) The effect of interference between circular cylinders in cross flow. J Fluids Struct $1: 239-261$

Zheng S, Zhang W, Lv X (2016) Numerical simulation of cross-flow around three equal diameter cylinders in an equilateral-triangular configuration at low Reynolds numbers. Comput Fluids 130:94-108

Publisher's Note Springer Nature remains neutral with regard to jurisdictional claims in published maps and institutional affiliations. 